\title{
The egg-shaped crystals that should not be: a brain-like texture combined with single crystallinity
}

\author{
Vivek Singh ${ }^{\dagger}$ Lothar Houben, ${ }^{\dagger}$ Linda J. W. Shimon, ${ }^{\dagger}$ Sidney R. Cohen $\stackrel{\dagger}{\dagger}$ \\ Ofra Golani, ${ }^{\S}$ Yishay Feldman, ${ }^{\dagger}$ Michal Lahav, ${ }^{\dagger}, *$ and \\ Milko E. van der Boom ${ }^{\dagger}, *$ \\ $\dagger$ Department of Molecular Chemistry and Materials Science, ${ }^{\dagger}$ Department of Chemical \\ Research Support, ${ }^{\S}$ Department of Life Sciences Core Facilities, Weizmann Institute of \\ Science, Rehovot 7610001, Israel. \\ Email: michal.lahav@weizmann.ac.il; milko.vanderboom@weizmann.ac.il
}

\begin{abstract}
We demonstrate here a unique metallo-organic material where the appearance and the internal crystal structure contradict. The egg-shaped (ovoid) crystals have a "brain-like" texture. Although these micro-sized crystals are monodispersed; like fingerprints their grainy surfaces are never exactly alike. Remarkably, our X-ray and electron diffraction studies unexpectedly revealed that these structures are single-crystals comprising a continuous coordination network of homochiral hexagonal and triangular channels. By applying different reaction conditions, a series of isostructural and monodispersed crystals were obtained having a monodomain appearance. These results indicate that families of isostructural crystals following the established rules for crystal growth contain hidden gems to be discovered with fascinating multidomain morphologies.
\end{abstract}




\section{Introduction}

The shape and morphology of crystals provide intuitive information on the growth process and crystallinity. ${ }^{1,2}$ However, many naturally occurring crystals have shapes which do not correlate with their crystallographic symmetry. Crystallization processes are known to produce hierarchical superstructures by the alignment of small crystallites. For example, biomineralization can afford materials with superior properties, complex morphologies and hierarchical order. ${ }^{3,4}$ Most of these natural materials are based on single crystals of calcium carbonate. The existence of specific and complex morphologies is a direct result of the evolutionary approach of nature and needed to fulfil various tasks. For example, coccoliths are composed of calcium carbonate which is known to be brittle. However, the interlocking architecture of coccoliths enhances significantly their damage tolerance. ${ }^{5-7}$ Another example is nacre formed by molluscs, a polycrystalline biomineral also based on calcium carbonate, that shows excellent mechanical properties. ${ }^{8,9}$

Whereas crystal engineering of molecular materials has focused mainly on control over the crystallographic structures, many excellent works demonstrate the use of additives and modulators to control crystal dimensions and morphology. ${ }^{10-13}$ For example, benzoic acid derivative has been used by Fischer et al to control the dimensions of metal-organic frameworks (MOFs). ${ }^{14}$ Recently, Yaghi and coworkers used aniline to control the dimensions of covalent organic frameworks (COFs). ${ }^{15}$ Nevertheless, the predesign and control of crystal morphology, uniformity and monodispersity is still a tremendous challenge. As a general rule, the crystal lattice of a singlecrystal is continuous and lacks grain boundaries. Such crystals have a monodomain appearance and their diffraction patterns consist of well-defined reflections. Only a few molecule-based crystals have been reported that exhibit paradoxically both single-crystallinity and a multidomain appearance. We reported recently two examples of such uncommon crystals. ${ }^{16,17}$ Their domains are relatively large relative to the overall sizes of the crystals. Our findings strengthen the case for new class of crystalline materials that combine structural and morphological properties which do not obviously co-exist. The rules to achieve such crystals are as of yet not entirely clear, as many parameters are likely to play a role including bond strength, nucleation, directional growth and stability. The evolution of multidomain surfaces on single-crystal cores from porous materials including MOFs, zeolites and porous carbon has not been widely explored. For example, MOF-74 forms polycrystalline aggregates which is also very common for minerals and inorganic 
crystals. ${ }^{18,19}$ The complex crystalline surface features are sensitive to the concentration and ratios of the building-blocks.

In this work, we report the growth and structure of egg-shaped single-crystals exhibiting a brain-like texture. The three-dimensional structure of the crystals was revealed using electron tomography combined with scanning electron diffraction (SED) experiments. These SED measurements unambiguously demonstrate that the brain-like texture does not contradict the single-crystallinity. The crystalline surface features are sensitive to the concentration and ratios of the building blocks (Scheme 1). The low monodispersity and egg-shaped structures belong to a series of isostructural crystals having a rare spacegroup (P622) and a monodomain-like crystalline surface.

\section{Results and Discussion}

We used a combination of a tetrahedral ligand (AdDB) ${ }^{20}$ having four pyridyl groups and a bivalent nickel salt to form our crystals. The structure of AdDB is such that multidentate binding of the pyridyl groups to one metal center is structurally prohibited; the binding of several metal centers to one ligand is a realistic possibility, as there are no structural or electronic reasons forbidding this. Coordination of pyridine-derivatives to divalent $\mathrm{NiX}_{2}(\mathrm{X}=$ anion) is known to result in 6-coordinated octahedral complexes.

Reacting AdDB with $\mathrm{Ni}\left(\mathrm{NO}_{3}\right)_{2} \cdot 6 \mathrm{H}_{2} \mathrm{O}$ under solvothermal conditions in a glass pressure vessel resulted in the egg-shaped crystals having a brain-like texture (Ni-AdDB). The starting materials were heated at $105{ }^{\circ} \mathrm{C}$ at a molar ratio of 1:2 in a mixture of dimethylformamide (DMF) and chloroform (1:3 v/v). Both AdDB and Ni( $\left.\mathrm{NO}_{3}\right)_{2} \cdot 6 \mathrm{H}_{2} \mathrm{O}$ have good solubility in this solvent mixture. After $48 \mathrm{~h}$, the reaction mixture was gradually cooled down to room temperature. Light green crystals were isolated in high yield (> 90\%) by centrifugation and subsequently washing with organic solvents. The reaction conditions allow possible kinetic products formed from rapid metalligand coordination to transform into thermodynamically favored structures. Octahedral nickel

complexes can undergo ligand dissociation: $\left[\mathrm{NiX}_{2} \mathrm{~L}_{2}\right]+2 \mathrm{~L} \rightarrow\left[\mathrm{NiX}_{2} \mathrm{~L}_{4}\right]$ with $\mathrm{L}$ being a neutral, monodentate ligand. ${ }^{21}$

Scanning electron microscope (SEM) imaging of Ni-AdDB revealed several interesting aspects: the crystals are highly uniform, both in their appearance and dimensions (Figure 1). Their egg shape is uncommon, and almost no fused crystals or other morphologies were observed. 
Analysis of low magnification SEM images of 100 crystals revealed average values for the major axis and minor axis of $1.6 \pm 0.1 \mu \mathrm{m}$ and $1.0 \pm 0.1 \mu \mathrm{m}$, respectively. High magnification SEM images reveal the unexpected textured surfaces and the apparently random arrangement of the distinct domains. These domains have well-defined boundaries separated by valleys, but vary in shape and size. Despite the similarities between the shapes and dimensions of the individual crystals, no two crystals have been found to have the same irregularly textured surface.

Atomic Force Microscopy (AFM) measurements and SEM-rendered AFM images confirm the unique texture of the crystals and provided additional information regarding the multidomain features of the surfaces (Figure 2A-B). The size and shape of the domains are indeed irregular and these domains are distributed over the entire particle. The domain boundaries are well-separated as demonstrated by the profile lines showing depths of a few nanometers (Figure S1). The elastic modulus of the crystals is $3.0 \pm 0.7 \mathrm{GPa}$, which is similar to other coordination-based polymers (e.g., ZIF-8). ${ }^{22,23}$

The shapes of a large number of domains from the center of $\sim 100$ crystals (to minimize possible distortion caused by curvation) were analyzed by imaging software using a code developed by us. ${ }^{24}$ We used Fiji macro and elastic boundary-based segmentation with multicut workflow to quantify the shapes of $\sim 2000$ domains (or small cluster of domains). A perfect convex shape or circle have solidity or circularity of 1 , respectively. The irregular subunits of the crystals have a mean solidity of $0.80 \pm 0.01$ indicating that certain parts have a convex shape (Figure 2C-H). The mean circularity of $0.60 \pm 0.02$ shows that the shapes cannot be classified as circular. The selected surface areas vary largely: from $200 \mathrm{~nm}^{2}$ to $0.4 \mu \mathrm{m}^{2}$.

The crystal shape and the brainy appearance are preserved during heating $\left(200{ }^{\circ} \mathrm{C}\right)$ in air for 4 hours as shown in SEM and AFM images (Figure S2, S3). No fusion of the domains was observed: the same crystals were observed before and after heating by AFM. The high thermal stability is also indicated by thermogravimetric analysis (TGA) under air (Figure S4). A total weight loss of $20 \%$ was observed upto $130^{\circ} \mathrm{C}$ attributed to the loss of solvents. The material decomposes between $215^{\circ} \mathrm{C}$ and $490{ }^{\circ} \mathrm{C}$ as indicated by a weight loss of $78 \%$. AdDB shows $98 \%$ weight loss between $277{ }^{\circ} \mathrm{C}$ and $636{ }^{\circ} \mathrm{C}$ (Figure S5). The morphological stability of Ni-AdDB is also preserved in water and common organic solvents (i.e., chloroform, DMF, DCM, ethanol) at room temperature for at least six months. Micro-Raman and FTIR spectroscopy provide direct evidence for the complexation of the $d^{8}$ metal centers with the vinyl-pyridine groups (Figure S6, S7). For example, 
the intensity of the ethylenic double bonds observed at $v=1635 \mathrm{~cm}^{-1}$ changed from very strong to medium upon coordination. A shift is observed in the FTIR spectra for the peaks assigned to these bonds from $v=1633 \mathrm{~cm}^{-1}$ (AdDB) to $1657 \mathrm{~cm}^{-1}$. The peaks for the pyridine ring vibration at $v=$ $1595 \mathrm{~cm}^{-1}$ are also shifted to $1608 \mathrm{~cm}^{-1}$. The presence of the nitrate anions is indicated by the peak at $v=1384 \mathrm{~cm}^{-1}$.

To determine the crystallographic structure of the small crystals of Ni-AdDB, we obtained larger crystals (Ni-AdDB') suitable for structural determination by single-crystal X-ray diffraction (vide supra). These larger crystals were obtained by reducing the concentration of both starting materials by one order of magnitude, while keeping all other parameters identical. SEM analysis revealed the formation of uniform crystals having an average size of $14.0 \pm 1.3 \mu \mathrm{m}$ (100 crystals) and a faceted hexagonal and a relative smooth surface (Figure S8).

Using Powder X-Ray Diffraction (PXRD) measurements (Figure 3A, B), we refined the crystal structure of Ni-AdDB, and found one crystal phase similar to the structure of Ni-AdDB'. The crystallographic packing of Ni-AdDB and the larger, morphologically different Ni-AdDB' are nearly identical as shown by the similarities between the experimental data and simulated PXRD spectrum of the single-crystal X-ray data of Ni-AdDB'. The estimated unit cell parameters of NiAdDB $(P 622, \mathrm{a}=25.6667 \AA, \mathrm{b}=25.6667 \AA$ and $\mathrm{c}=17.9702)$ are very close to the single-crystal data values for Ni-AdDB' $(P 622, a=25.8953 \AA, b=25.8953 \AA$ and $\mathrm{c}=17.8576)$ (Figure 3C, Figure S9). Using the broadening of the peaks, the coherent scattering length was estimated from the Williamson-Hall plots for Ni-AdDB and Ni-AdDB' at $20-30 \mathrm{~nm}$ and $\sim 130 \mathrm{~nm}$, respectively. The small coherent scattering length of Ni-AdDB most likely corresponds to the size of the surface domains as observed by SEM and AFM.

The continuous framework consists of divalent metal centers having an octahedral, chiral geometry. The unit cells consist of six metal centers. The metal cations are bound to the pyridine groups of four different ligands in the equatorial positions forming a chiral, propeller-type arrangement possibly to minimize the steric hindrance between adjacent ligands. ${ }^{25,26}$ The four Ni$\mathrm{N}$ bond distances are within the range of 2.073-2.086 $\AA$. The coordination chemistry resulted in two different helical 1D structures: trigonal (three fold) and a hexagonal (six fold) channels are prominent structural features. Each hexagonal channel is surrounded by six trigonal channels. The diameter of the hexagonal and triangular pores is $\sim 7.7 \AA$ and $\sim 10.2 \AA$, respectively. 
The single crystallinity of Ni-AdDB was demonstrated by a series of scanning electron diffraction (SED) experiments on individual crystals (Figure 4A-E). The experimental conditions required the use of lose-dose exposure in combination with Cryo-preservation. The results of these SED experiments and the single-crystal X-ray diffraction measurements of Ni-AdDB' also show that these morphologically different crystals have similar crystallographic structures. This observation is in good agreement with the bulk PXRD data (vide supra). SED patterns extracted from the marked regions of interest for Ni-AdDB (Figure 4B, C1-4), and simulated diffraction patterns of Ni-AdDB' show the same zone axis, <120> (Figure 4D). The strong Bragg diffraction of another particle also matches well with the simulated electron diffraction pattern of Ni-AdDB' in the viewing direction $\langle 150\rangle$ (Figure 4B, C5, E). The observed diffraction patterns result from crystalline areas close to the surface ( 100 nanometers depth) and from the core. This observation proves the single-crystal nature of Ni-AdDB and the similarity between the crystallographic structures. These observations imply that growth mechanisms do not involve the addition of amorphous material to the crystal surface followed by secondary nucleation. Such a mechanism would typically result in domain structures with multiple orientations, in contradiction with the diffraction data. Alternatively, epitaxial growth can occur with crystalline re-ordering from an amorphous to crystalline state. Such a mechanism is in agreement with follow-up SEM experiments showing already at the initial growth stages the presence of domains, clefts, and eggshape structures (vide infra).

A 3D-reconstruction of Ni-AdDB was generated using electron tomography (Figure 4F-I). This method allows us to visualize the inner structural features of an individual crystal. The sample is stepwise $\left(2^{\circ}\right)$ tilted from $-50^{\circ}$ to $+50^{\circ}$ during the measurements allowing the observation of $3 \mathrm{D}$ structural features. A representative image of a crystal measured at a tilt angle of $26^{\circ}$ is shown in Figure 4F and growth islands are shown in a volume projection image (Figure 4G). An orthoslice close to the surface also shows similarly shaped growth islands and facets (Figure $\mathbf{4 H}$ ). These facets are also observed in a cross-section along the major axis (Figure 4I). A series of crosssections perpendicular and along the major axis is shown in the movie $\mathbf{S 1}$ further confirming the presence of well-faceted structures inside the crystal.

SEM and TEM measurements at the initial stages of the formation of Ni-AdDB revealed the formation of two different kinds of structures $(t=2 \mathrm{~min}$; $1-4 \mu \mathrm{m}$ from TEM; Figure 5A, Movie S2). Electron diffraction measurements revealed that these structures are crystalline and already 
have a crystallographic packing similar to Ni-AdDB. SED measurements show that the plate-like sub-units (thickness 50-200 nm) of the larger structures are oriented and all have the same habit planes (Figure S10, S11). After $5 \mathrm{~min}$, egg-shaped structures having a grainy texture are already observable and resemble the crystal morphology and monodispersity observed after prolonged heating (Figure 5B-D). During the solvothermal reaction, these structures increase in size (5 min. minor axis $=0.6 \pm 0.2 \mu \mathrm{m}$, major axis $=0.8 \pm 0.3 \mu \mathrm{m} ; 2$ days: minor axis $=1.0 \pm 0.1 \mu \mathrm{m}$, major axis $=1.6 \pm 0.1 \mu \mathrm{m})($ Figure S12). Time-dependent PXRD measurements (5 min-48 h) show that during the growth the bulk crystallinity is retained (Figure S13).

Interestingly, the surface texture and crystal dimensions are clearly a function of the concentrations employed and the stoichiometry of the building blocks. The SEM results show that the unique brain-like appearance of Ni-AdDB is sensitive to the supersaturation (Figure 6 top, Figure S14). Doubling the concentrations of both building blocks resulted in smaller, sub-micrometer sized (300-500 nm) crystals having a cylindrical morphology with a smooth surface texture. A five-fold increase of the concentrations resulted in the formation of hexagonal prism (100-200 nm) having very smooth, featureless surfaces. Decreasing the concentrations (two- and five-fold) resulting in oval shaped structures (length: 9-10 $\mu \mathrm{m}$ ) with pointed tips, and structures having a hexagonal morphology (length: 16-17 $\mu \mathrm{m}$ ), respectively. It is known that the concentration can affect the dimensions of crystals. ${ }^{27}$ In our case, we also observe striking differences in morphology and surface texture. The uniformity of the eggshaped crystals for a given concentration is high. PXRD measurements showed that the concentration of the starting materials does not seemingly affect the crystallographic structure, all these morphologically different crystals belong to the rare space group of $P 622$. The stoichiometry of the building blocks was also systematically varied within the range of $\mathrm{Ni}\left(\mathrm{NO}_{3}\right)_{2} \cdot 6 \mathrm{H}_{2} \mathrm{O}:$ AdDB $=0.3: 1-6: 1$. We observed the fascinating multidomain surface by using a metal-to-ligand ratio of 2:1(Figure 6, bottom, Figure S15). Excess of AdDB results in a smooth surface and larger crystals. Excess of the metal salt afforded smaller crystals indicating a faster nucleation process. The crystallographic structures remain the same regardless of the reaction conditions (Figure S16-S17). These observations suggest a growth process where the reactivity of crystal surfaces is affected by binding of the excess of one of the molecular building blocks. Therefore, X-ray photoelectron spectroscopy (XPS) was used to analyze the metal ion content at the surfaces of the crystals obtained by varying the metal-to-ligand 
ratio (Figure 6 bottom, S15, S18). The nickel cations retained their oxidation state $(+2)$ as judged by the binding energy of $855.8 \mathrm{eV}$ for $\mathrm{Ni} 2 \mathrm{p}^{3} / 2$ and the satellite bands (859-869 eV and 876-886 $\mathrm{eV})$. We also observed a signal at $407 \mathrm{eV}$, which can be attributed to the counterions $\left(\mathrm{NO}_{3}{ }^{-}\right)$. The $\mathrm{N}_{\text {pyr }} / \mathrm{Ni}^{2+}$ ratio decreases from 3.5 to 2.7 upon increasing the metal-to-ligand ratio from $0.3: 1-$ 6:1. For a fully formed network a $\mathrm{N}_{\mathrm{pyr}} / \mathrm{Ni}^{2+}$ ratio of 4 is expected. A surface saturated with nickel cations, only partly bound to the pyridine groups would reduce this value. The results are in good agreement with the hypothesis that the use of an excess of the metal salt results in a growth process whereby the developing crystal surface is covered with metal cations. Hence, the formation of different morphologies when different ratios of the building blocks are used.

\section{Conclusions}

An unexpected finding of our study is the unusual crystal appearance (i.e., shape and texture) combined with single crystallinity. Randomly oriented and irregularly shaped domains form a brain-like surface texture without perturbation of the single-crystal nature. The internal structure of these crystals consists of plate-like growth islands. Time-dependent measurements show that at the initial stage of the reaction the crystallographic structure is already formed, whereas the crystals undergo a remarkable morphological transformation. Plate-like crystals transform rapidly into egg-shaped structures having a brain-like surface texture. These newer structures increase in size while maintaining a high level of uniformity. Regardless of the reaction conditions (time, concentrations, and ratios of the molecular building blocks), the crystallographic structures of the twelve morphologically different crystals are very similar with the achiral molecules giving homochiral, continuous channels. The emergence of chirality in molecular materials, including crystals as observed in this study, from achiral precursors is a fascinating and complex phenomenon that is often related to origin of life studies. ${ }^{28-33}$ Interestingly, we have found several isostructural and homochiral crystals having the same spacegroup (P622) but a wide range of morphologies and sizes. To this end, the coordination chemistry of metal-pyridine systems offers opportunities to generate fascinating mono- and multidomain, homogeneous single crystals.

\section{Acknowledgements}

This research was supported by the Irving and Cherna Moskowitz Center for Nano and Bioimaging at the Weizmann Institute of Science, the Israel Science Foundation (ISF), the Minerva 
Foundation and the Weizmann-Yale collaborative program. We thank Dr Tatyana Bendikov (WIS) for the XPS measurements, and the scientists of ID-29 beamline at ESRF (Grenoble, France) for their technical support. We thank the staff of Rigaku, in particular Dr Jakub Wojciechowski. We would also like to thank Prof. J. M. McBride (Yale University) for the fruitful discussions. M.E.v.d.B. holds the Bruce A. Pearlman Professional Chair in Synthetic Organic Chemistry. V.S. is the recipient of a Sustainability and Energy Research Initiative (SAERI) PhD fellowship.

\section{References}

1. Addadi, L., and Weiner, S. (1992). Control and design principles in biological mineralization. Angew. Chem. Int. Ed. 31, 153-169.

2. Donnay, G., and Pawson, D. L. (1969). X-ray diffraction studies of echinoderm plates. Science 166, 1147-1150.

3. Colfen, H., and Antonietti, M. (2005). Mesocrystals: Inorganic superstructures made by highly parallel crystallization and controlled alignment. Angew. Chem. Int. Ed. 44, 55765591.

4. Mann, S. (1955). Molecular recognition in biomineralization. Nature, 332, 119-124.

5. Jaya, B. N., Hoffmann, R., Kirchlechner, C., Dehm, G., Schen, C., and Langer, G. (2016). Coccospheres confer mechanical protection: New evidence for an old hypothesis. Acta Biomater. 42, 258-264.

6. Lomora, M., Shumate, D., Rahman, A. A., and Pandit, A. (2019). Therapeutic applications of phytoplankton, with an emphasis on diatoms and coccolithophores. Adv. Ther. 2, 1-24.

7. Surfaces and Interfaces for Biomaterials Woodhead Publishing Series in Biomaterials 2005, 666-692.

8. Nudelman, F., and Sommerdijk, N. (2012). Biomineralization as an inspiration for materials chemistry. Angew. Chem. Int. Ed. 51, 6582-6596.

9. Rosenhek-Goldian, I., and Cohen, S. R. (2020). Nanomechanics of biomaterials - from cells to shells. Isr. J. Chem. 60, 1171-1184.

10. Desiraju, G. R. (2013). Crystal engineering: From molecule to crystal. J. Am. Chem. Soc. 135, 9952-9967.

11. Hollingsworth, M. D. (2002). Crystal engineering: from structure to function. Science 295, 2410-2413.

12. Weissbuch, I., Addadi, L., Lahav, M., and Leiserowitz, L. (1991). Molecular recognition at crystal interfaces. Science 253, 637-645.

13. Xu, H. S., Luo, Y., Li, X., See, P. Z., Chen, Z. X., Ma, T. Q., Liang, L., Leng, K., Abdelwahab, I., Wang, L., Li, R. L., Shi, X. Y., Zhou, Y., Lu, X. F., Zhao, X. X., Liu, C. B., Sun, J. L., and Loh, K. P. (2020). Single crystal of a one-dimensional metallo-covalent organic framework. Nat. Comm. 11, 1434.

14. Hermes, S., Witte, T., Hikov, T., Zacher, D., Bahnmuller, S., Langstein, G., Huber, K., and Fischer, R. A. (2007). Trapping metal-organic framework nanocrystals: An in-situ time- 
resolved light scattering study on the crystal growth of MOF-5 in solution. J. Am. Chem. Soc. 129, 5324-5325.

15. Ma, T. Q., Kapustin, E. A., Yin, S. X., Liang, L., Zhou, Z. Y., Niu, J., Li, L. H., Wang, Y. Y., Su, J., Li, J., Wang, X. G., Wang, W. D., Wang, W., Sun, J. L., and Yaghi, O. M. (2018). Single-crystal X-ray diffraction structures of covalent organic frameworks. Science 361, 48-52.

16. di Gregorio, M. C., Shimon, L. J. W., Brumfeld, V., Houben, L., Lahav, M., and van der Boom, M. E. (2020). Emergence of chirality and structural complexity in single crystals at the molecular and morphological levels. Nat. Commun. 11, 380 .

17. di Gregorio, M. C., Elsousou, M., Wen, Q., Shimon, L., Brumfeld, V., Houben, L., Lahav, M., and van der Boom, M. E. (2021). Molecular cannibalism: Sacrificial materials as precursors for hollow and multidomain single crystals. Nat. Commun. 12, 957.

18. Shtukenberg, A. G., Punin, Y. O., Gunn, E., and Kahr, B. (2012). Spherulites. Chem. Rev. 112, 1805-1838.

19. Feng, L., Wang, K. Y., Yan, T. H., and Zhou, H. C. (2020). Porous crystalline spherulite superstructures. Chem 6, 460-471.

20. Neumann, T., Jess, I., Nather, C. (2018). Crystal structures of (acetonitrile-kappa N)tris(pyridine-4-thioamide-kappa N)bis(thiocyanato-kappa N)cobalt(II) acetonitrile disolvate and tetrakis(pyridine-4-thioamide-kappa N)bis(thiocyanato-kappa N)nickel(II) methanol pentasolvate. Acta Crystallographica Section E-Crystallographic Communications, 74, 964.

21. Wilkins, R. G. (1970). Mechanisms of ligand replacement in octahedral nickel(II) complexes. Acc. Chem. Res. 3, 408-416.

22. Redfern, L. R., and Farha, O. K. (2019). Mechanical properties of metal-organic frameworks. Chem. Sci. 10, 10666-10679.

23. Tiba, A., Tivanski, A. V., and MacGillivray, L. R. (2019). Size-dependent mechanical properties of a metal-organic framework: increase in flexibility of ZIF-8 by crystal downsizing. Nano Lett. 19, 6140-6143.

24. https://github.com/WIS-MICC-CellObservatory/BrainyCrystals/

25. Hamm, D. J., Bordner, J., and Schreiner, A. F. (1973). The crystal and molecular structure of diiodotetrakis(pyridine)nickel(II) [Nipy ${ }_{4} \mathrm{I}_{2}$ ]. Inorg. Chim. Acta 7, 637-641,

26. Wöhlert, S., Wriedt, M., Jess, I., and Näther, C. (2012). Bis(dicyanamido$\kappa \mathrm{N})$ tetrakis(pyridine-- $\mathrm{NN}$ )nickel(II). Acta Cryst. Sect. E 68, E68- m745.

27. Wang, S. Z., McGuirk, C. M., d'Aquino, A., Mason, J. A., and Mirkin, C. A. (2018). MetalOrganic Framework Nanoparticles. Adv. Mat. 2018, 30.

28. Vogl, O. (2011). Chiral crystallization and the origin of chiral life on earth. J. Polym. Sci., Part A-1: Polym. Chem. 49, 1299-1308.

29. Morris, R. E. and Bu, X. H. (2010). Induction of chiral porous solids containing only achiral building blocks. Nature Chemistry 2, 353-361.

30. Soai, K., Kawasaki, T., and Matsumoto, A. (2019). Role of asymmetric autocatalysis in the elucidation of origins of homochirality of organic compounds. Symmetry (Basel). 11, 120. 
31. Pramanik, S., Kauffmann, B., Hecht, S., Ferrand, Y., and Huc, I. (2021). Light-mediated chiroptical switching of an achiral foldamer host in presence of a carbohydrate guest. Chem. Commun. 57, 93-96.

32. Viedma, C. (2005). Chiral symmetry breaking during crystallization: Complete chiral purity induced by nonlinear autocatalysis and recycling. Phys. Rev. Lett. 94, 3-6.

33. Greenfield, J.L., Evans, E.W., Di Nuzzo, D., Di Antonio, M., Friend, R.H., and Nitschke, J.R. (2018). Unraveling Mechanisms of Chiral Induction in Double-Helical Metallopolymers. J. Am. Chem. Soc. 140, 10344-10353.

34. Ophus, C. (2019). Four-Dimensional Scanning Transmission Electron Microscopy (4DSTEM): From Scanning Nanodiffraction to Ptychography and Beyond. Microsc. Microanal., 563-582.

35. Eggeman, A.S. (2019). Scanning transmission electron diffraction methods. Acta Crystallogr. Sect. B Struct. Sci. Cryst. Eng. Mater. 75, 475-484. 


\section{Figure captions}

Scheme 1. Complex morphologies including single crystallinity combined with a "brain-like" texture. (A) Solvothermal synthesis crystals using an achiral organic ligand (AdDB) with nickel(II) nitrate. (B) Left: multidomain crystal surface, right: scanning electron diffraction showing single crystallinity. (C) Control of dimensions, morphology and surface texture.

Figure 1. Morphological analysis of the brainy crystals using scanning electron microscopy (SEM). (A) Low magnification SEM image of Ni-AdDB, (B-C) High magnification SEM images of Ni-AdDB, and (D) Size distribution (100 crystals) of the major and minor axes of Ni-AdDB.

Figure 2. Surface details of Ni-AdDB by atomic force microscopy (AFM) and shape descriptions of surface domains. (A-B) SEM rendered AFM image and topographic image of Ni-AdDB, (C, F) SEM rendered AFM image and SEM image of Ni-AdDB respectively, (D, E) color coded circularity and solidity map of domains marked in $\mathrm{C}$ and $(\mathrm{G}, \mathrm{H})$ color coded circularity and solidity map of domains marked in F.

Figure 3. Crystallographic framework similarity between Ni-AdDB' and Ni-AdDB using powder X ray diffraction and helical channels in Ni-AdDB' framework. (A) X-ray diffraction comparison of Ni-AdDB (top), hexagonal crystals used for single crystal X-ray diffraction (NiAdDB'; middle) and simulated powder pattern from single crystal X-ray structure of hexagonal crystal Ni-AdDB' (bottom). (B) The experimental PXRD spectra (black line) of Ni-AdDB was fitted (red line) with the single crystal X-ray data of Ni-AdDB' (CCDC: 2020813) by the Whole Pattern Fitting (Pawley method) using unit cell parameters of Ni-AdDB'. The difference between the fitted and experimental curves is reported at the bottom of the two spectra (blue line). The base line is denoted in grey. The goodness of fit is $1.39(\mathrm{R} / \mathrm{E})$. (C) Top: crystallographic packing of hexagonal crystals (Ni-AdDB'), bottom: homochiral triangular and hexagonal channels.

Figure 4. Single crystallinity of Ni-AdDB using Cryo-scanning electron diffraction (SED) and 3D-reconstruction by electron tomography. The SED data was obtained in a 4D STEM approach. $^{34,35}$ (A) Virtual annular dark-field (ADF) image, i.e. the integral high angle scattering signal as a function of raster position. (B) Color-coded map of the correlation of each diffraction 
signal with a two-fold symmetric signal, indicating the spatial origin of pairs of crystalline Bragg reflections. Red means presence of strong Bragg diffraction with Bragg reflection pairs, blue means absence of or weak Bragg diffraction. Strong Bragg diffraction is observed in grazing incidence on the surface of the Ni-AdDB. In the interior the crystalline diffraction is washed out due to thickness-related inelastic background. (C) Diffraction patterns extracted from the regions of interest marked in (B). (D-E) Matching simulated electron diffraction pattern in the $\langle 120\rangle$ and $<150>$ uvw zone axis, based on the structure obtained from X-ray refinement. Marked regions 14 show the same zone axis and proves that Ni-AdDB is a single crystal. (F) ADF STEM image taken from a tilt series of images. The contrast is inverted for display. Contours disclose facets and a structure made out of patches. (G) Tomogram voxel projection accentuating growth islands of hexagonal shape. (H) Coronal orthoslice close to the surface of the ellipsoid. (I) Axial orthoslice close to the tip of the ellipsoid.

Figure 5. Morphological evolution of Ni-AdDB with time. (A) Plate-like morphologies after 2 min. (B-D) Egg-shaped structures after $5 \mathrm{~min}, 20 \mathrm{~min}$ and $48 \mathrm{~h}$ reaction time.

Figure 6. Relationships between crystal architecture, supersaturation, and ratios of building blocks. Top panel: SEM images of crystals obtained using different supersaturations with a 2:1 ratio of $\mathrm{Ni}\left(\mathrm{NO}_{3}\right)_{2} \cdot 6 \mathrm{H}_{2} \mathrm{O}$ and AdDB. Bottom panel: SEM images of crystals obtained using different ratios between $\mathrm{Ni}\left(\mathrm{NO}_{3}\right)_{2} \cdot 6 \mathrm{H}_{2} \mathrm{O}$ and AdDB. 


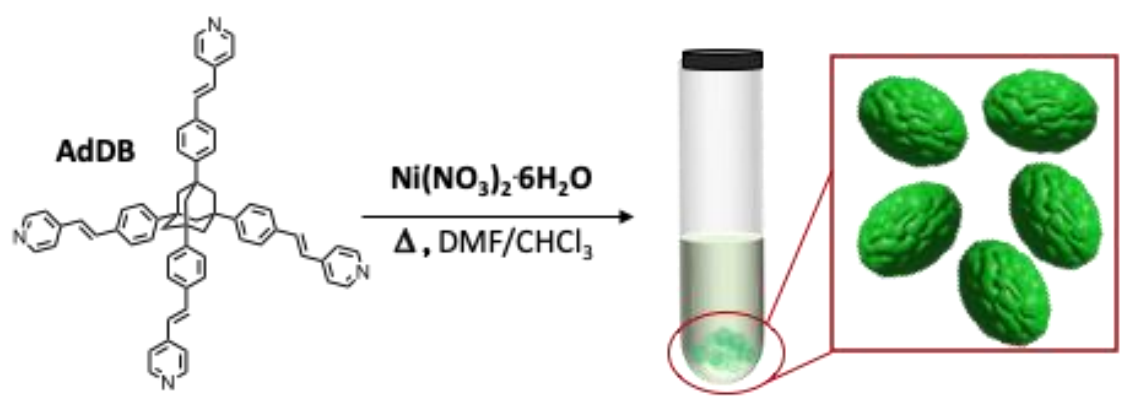

\section{Electron diffraction}

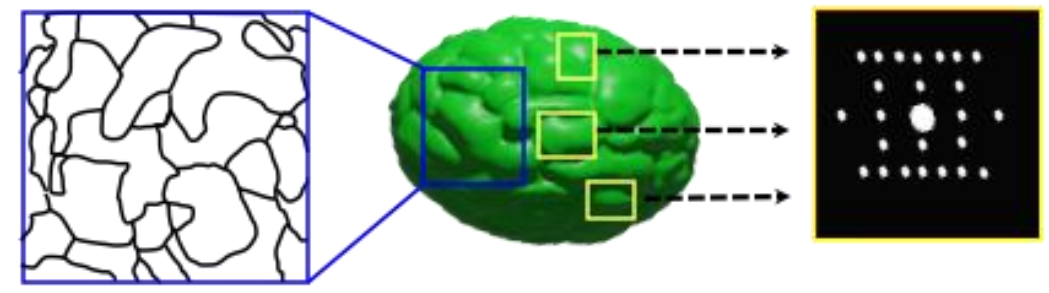

MULTIPLE DOMAINS

SINGLE CRYSTAL
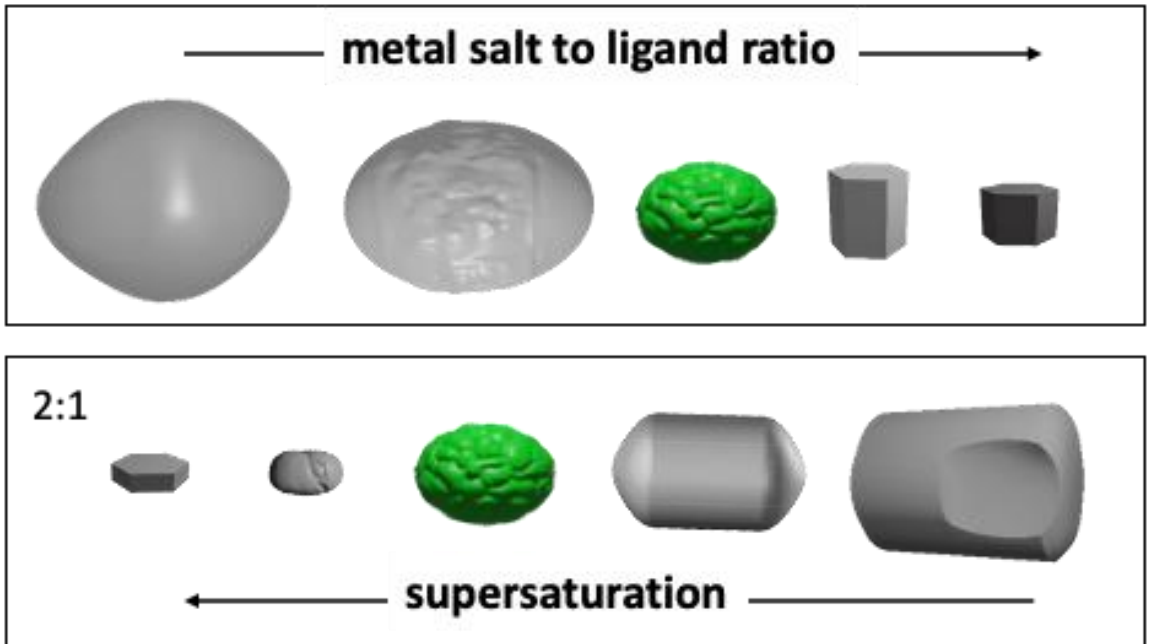


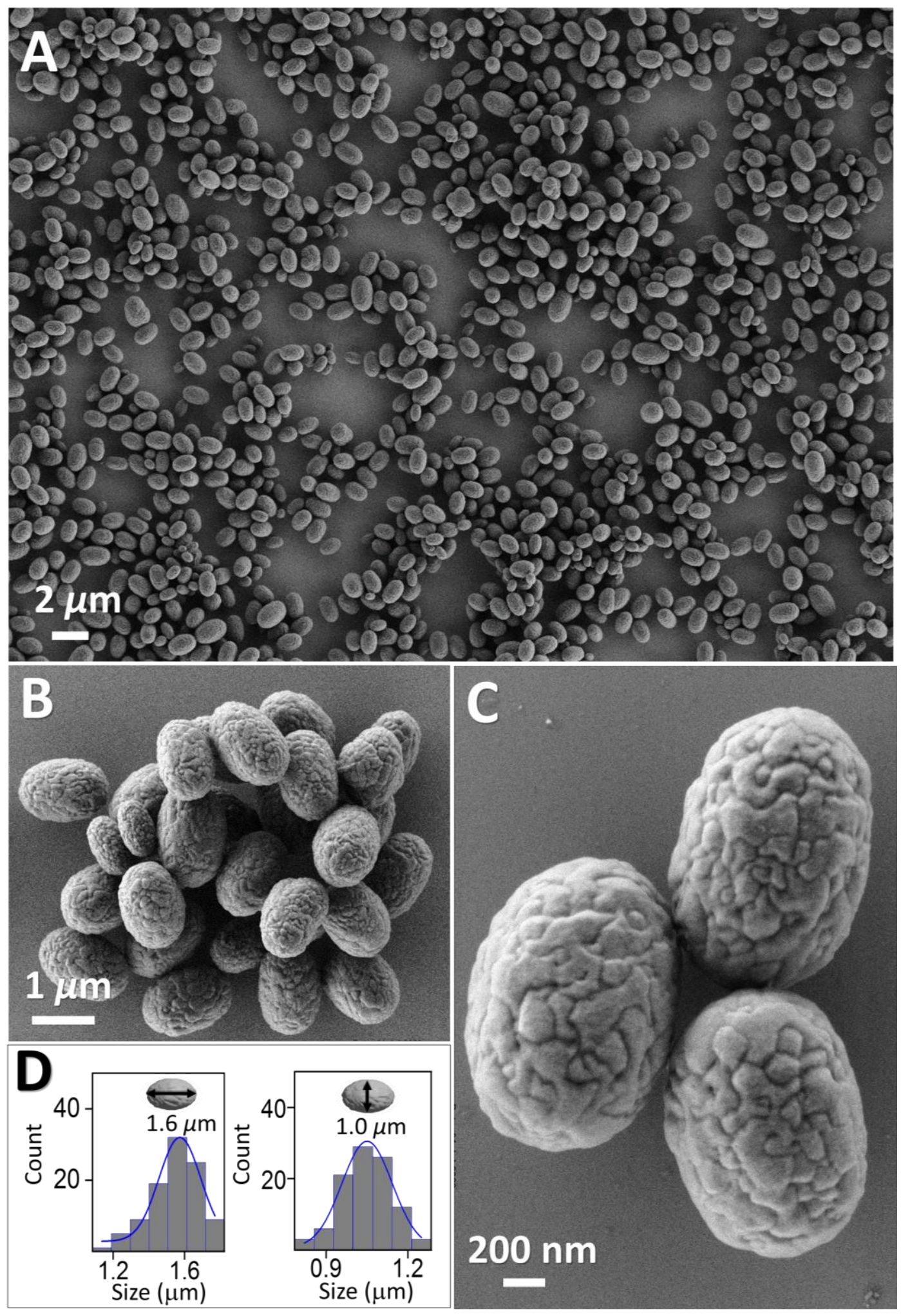




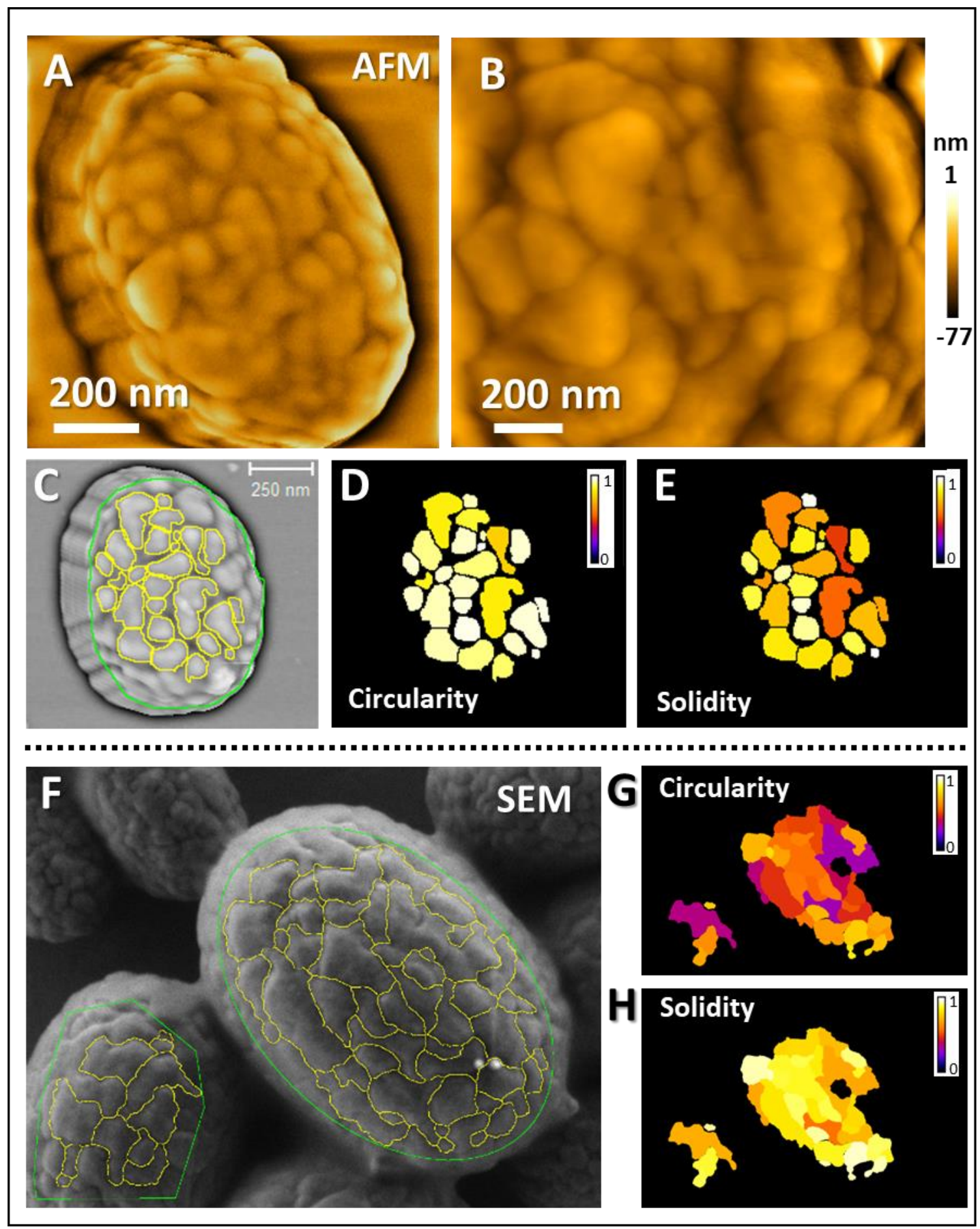



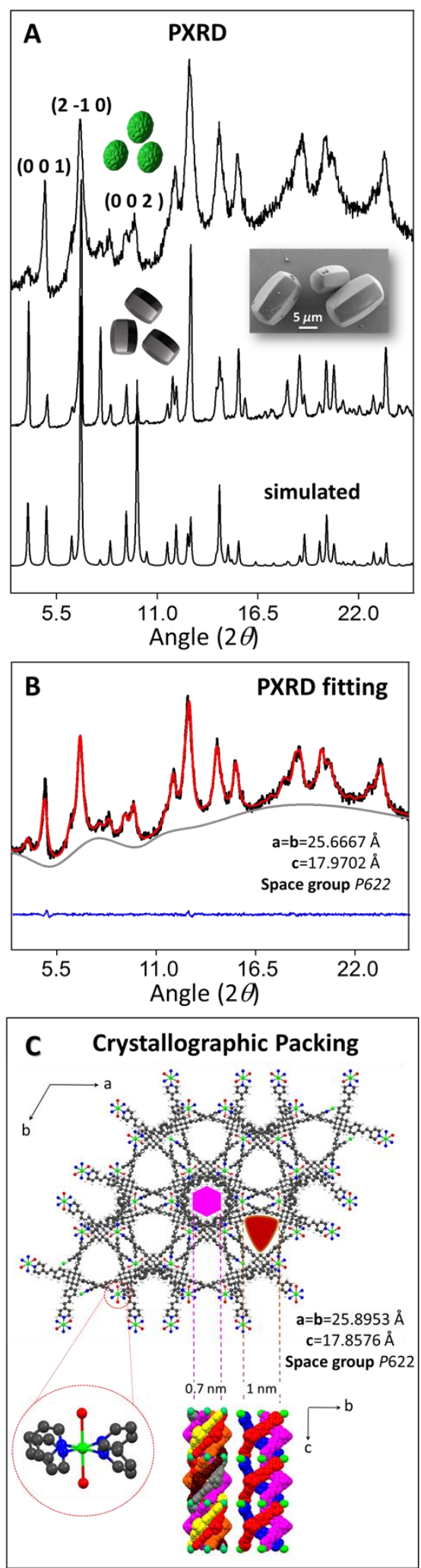


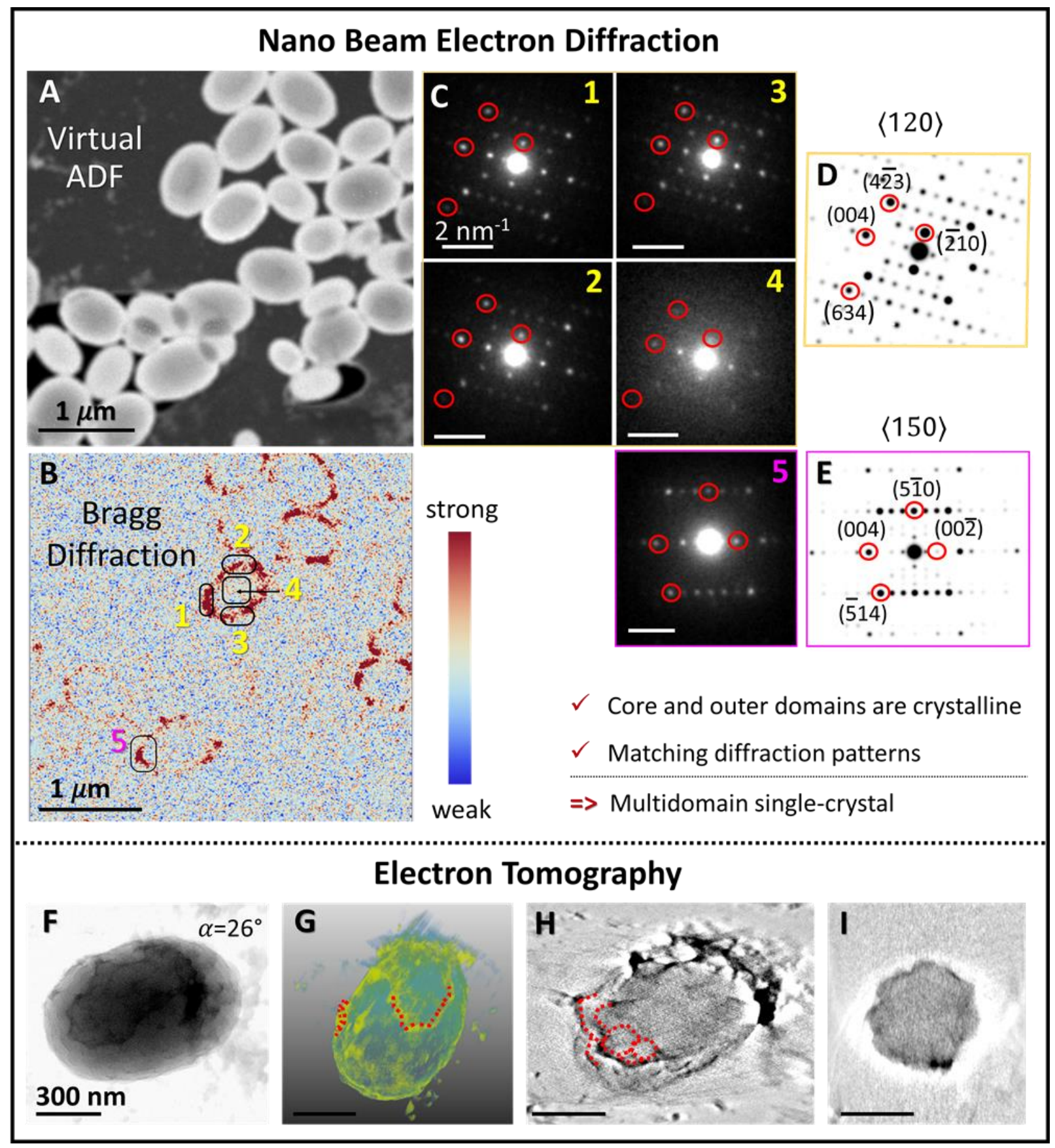



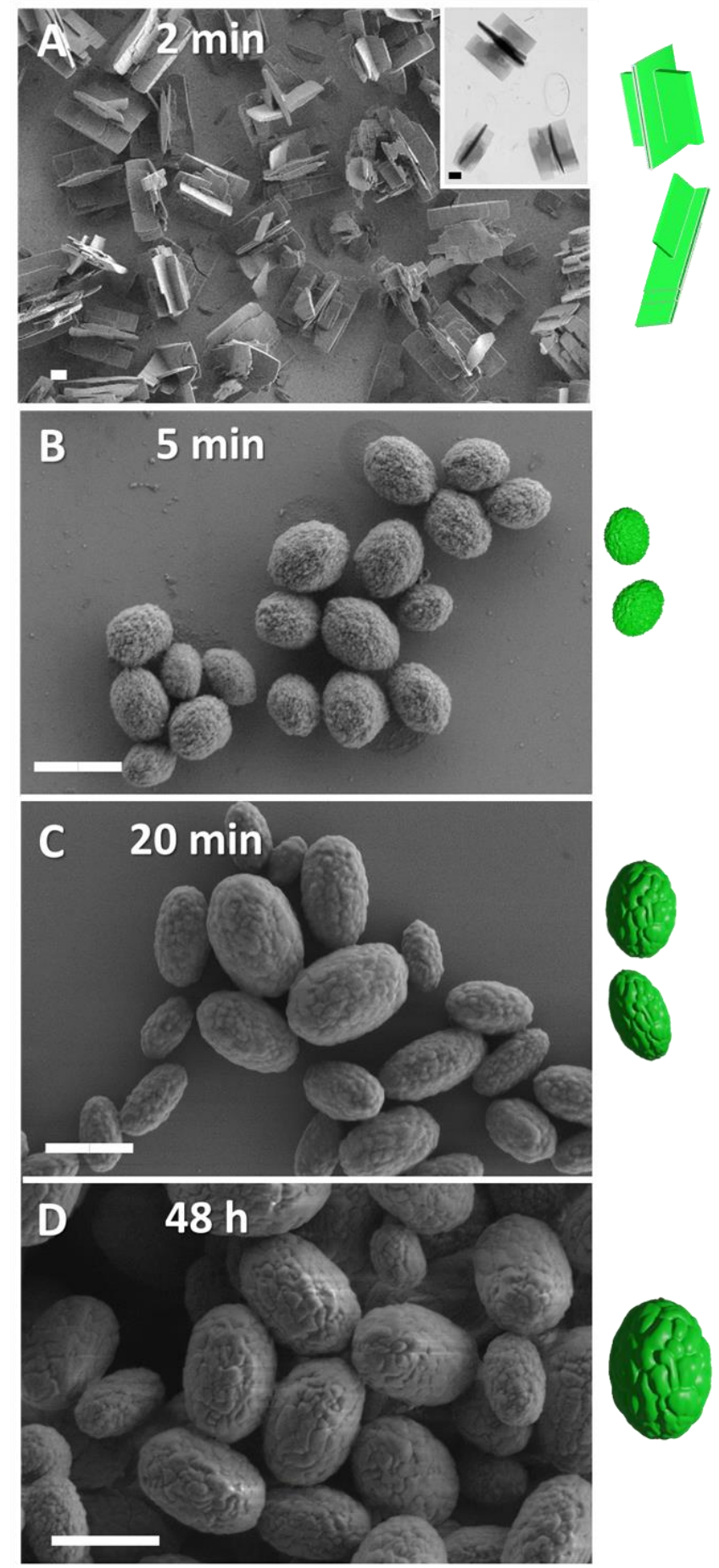

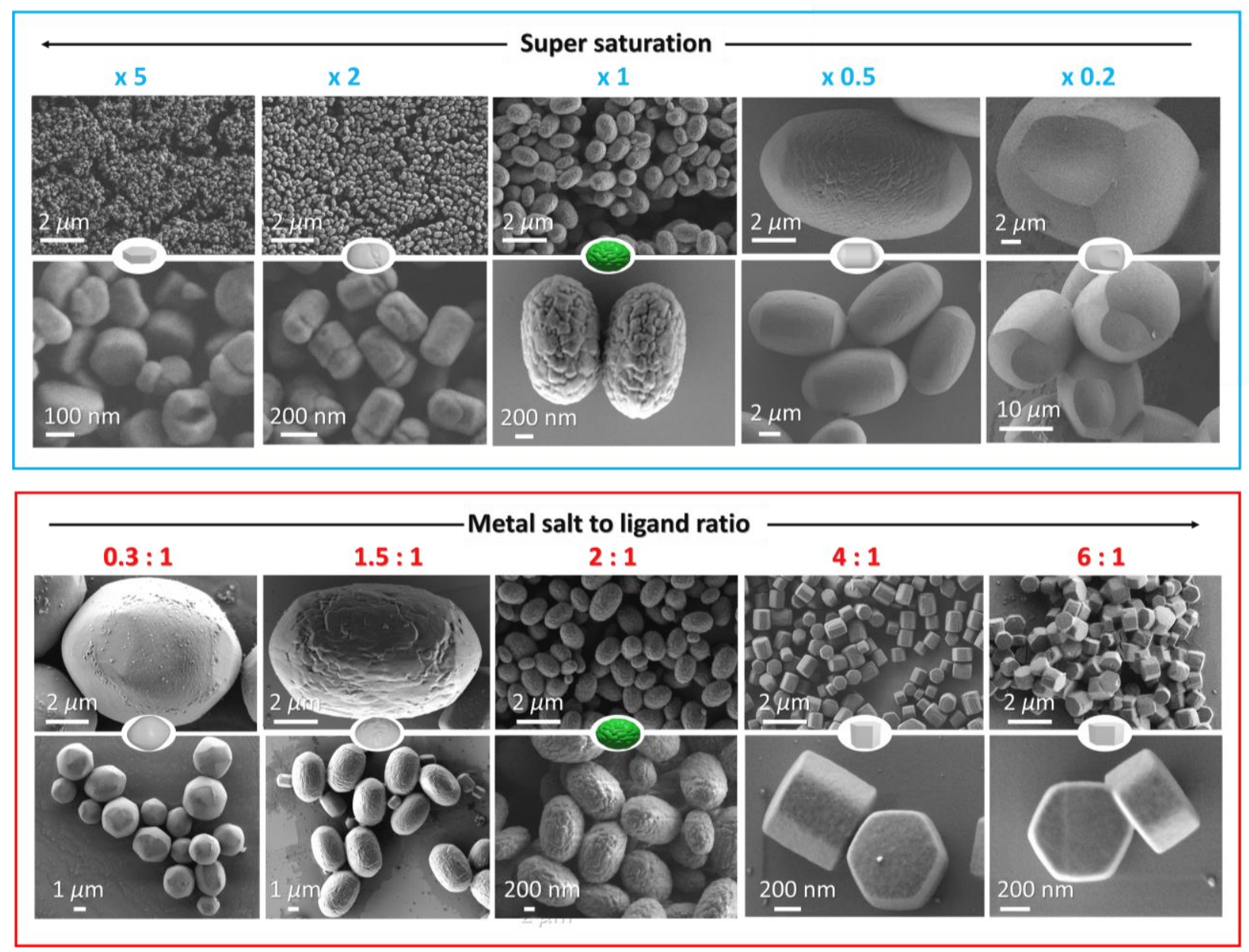


\title{
Supporting Information
}

\section{The egg-shaped crystals that should not be: a brain-like texture combined with single crystallinity}

\author{
Vivek Singh ${ }^{\dagger}$ Lothar Houben ${ }^{\dagger}$ Linda J. W. Shimon $\stackrel{\dagger}{\dagger}$ Sidney R. Cohen,${ }^{\dagger}$ Ofra \\ Golani, ${ }^{\S}$ Yishay Feldman, ${ }^{\dagger}$ Michal Lahav, ${ }^{\dagger}, *$ and \\ Milko E. van der Boom ${ }^{\dagger}, *$ \\ $\dagger$ Department of Molecular Chemistry and Materials Science $\$$ Department of Chemical \\ Research Support, ${ }^{\S}$ Department of Life Sciences Core Facilities, Weizmann Institute of \\ Science, Rehovot 7610001, Israel. \\ Email: michal.lahav@weizmann.ac.il; milko.vanderboom@weizmann.ac.il
}

\section{Materials and Methods}

Chloroform $\left(\mathrm{CHCl}_{3} \geq 99.8 \%\right)$ and dimethylformamide (DMF $\left.\geq 99.8 \%\right)$ were purchased from J. T. Baker and Sigma Aldrich, respectively. $\mathrm{Ni}\left(\mathrm{NO}_{3}\right)_{2} \cdot 6 \mathrm{H}_{2} \mathrm{O}(>99.9 \%)$ was purchased from Fluka and Sigma Aldrich. Reagents were used without further purification. 1,3,5,7-Tetrakis(4((E)-2-(pyridin-4-yl)vinyl)phenyl)adamantane (AdDB) was prepared according to a known

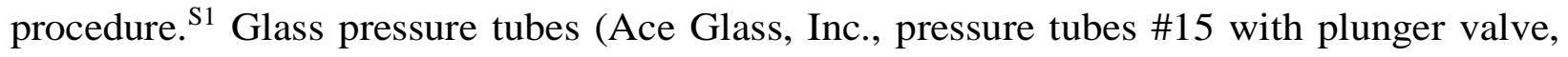
PTFE Bushing and FETFE® O-Ring, volume $50 \mathrm{~mL}$ ) were cleaned by a piranha solution for $10 \mathrm{~min}$ and then washed with deionized (DI) water and dried in an oven for $12 \mathrm{~h}$ at $130^{\circ} \mathrm{C}$. The infrared spectra were obtained using a Nicolet 460 single beam Fourier transform infrared spectrophotometer (FT-IR). Thermogravimetric analysis (TGA) was performed on a SDT Q600 V8.3 Build 101 instrument using alumina sample pans under a stream of air, heating at a rate of $10^{\circ} \mathrm{C} / \mathrm{min}$ between 25 and $1000^{\circ} \mathrm{C}$.

Formation of Ni-AdDB. Solutions of AdDB $(5.0 \mathrm{mg}, 5.9 \mu \mathrm{mol})$ in $\mathrm{CHCl}_{3}(1.0 \mathrm{~mL})$ and $\mathrm{Ni}\left(\mathrm{NO}_{3}\right)_{2} \cdot 6 \mathrm{H}_{2} \mathrm{O}(3.4 \mathrm{mg}, 12 \mu \mathrm{mol})$ in DMF $(3.0 \mathrm{~mL})$ were mixed in a glass pressure tube resulting in a clear-solution. The sealed tube was placed in an oven at $105^{\circ} \mathrm{C}$. After $48 \mathrm{~h}$, light green crystals 
were found at the bottom of the tube, the tube was removed from the oven and allowed to attain room temperature. These crystals were collected after centrifugation for $15 \mathrm{~min}$. at $4000 \mathrm{rpm}$, decanting the mother liquor and washing with ethanol $(2 \times 5 \mathrm{~mL})$. The crystals were dried using high vacuum for $5 \mathrm{~h}$ and obtained in near quantitative yield (>90\%).

Atomic Force Microscopy (AFM). AFM topographic measurements were made using a Bruker MMAFM in tapping mode with an AC160 probe (Olympus). Measurement conditions: tapping free amplitude $>30 \mathrm{~nm}$, setpoint ratio $>0.9$ and tip speed slow ( $\sim \mu \mathrm{m}$ per second) to obtain best resolution while avoiding sample degradation. Mechanical measurements were made on a JPK Nanowizard III in QI mode using an RTESP300 probe (Bruker). The sensitivity and spring constant were determined using JPK software. Force images were acquired at maximum applied force of 50-70 nN, collecting over 1000 pixel measurements on each image. The force maps were converted to modulus maps using the JPK software and Herzian analysis, rejecting any values above $22 \mathrm{GPa}$ which were obtained on most of the substrate pixels and some of the border regions. The statistics were obtained from the modulus maps by selecting the region of the MOF with a mask and computing mean and standard deviation with Gwyddion software, also used for rendering of the images. ${ }^{\mathrm{S} 2}$ The tip radius was determined by performing a similar analysis on a sample of polystyrene with known modulus.

Scanning electron microscopy (SEM). SEM measurements were performed using HRSEM Zeiss Sigma 500 and Zeiss Supra 55 at an EHT voltage of 1-3 kV. The samples were prepared by placing a drop of the reaction mixture on silicon substrates which were dried under air.

Single crystal X-ray diffraction of Ni-AdDB'. Crystals were obtained as follows: solutions of AdDB $(0.5 \mathrm{mg}, 0.6 \mu \mathrm{mol})$ in $\mathrm{CHCl}_{3}(1.0 \mathrm{~mL})$ and $\mathrm{Ni}\left(\mathrm{NO}_{3}\right)_{2} \cdot 6 \mathrm{H}_{2} \mathrm{O}(0.3 \mathrm{mg}, 1.2 \mu \mathrm{mol})$ in DMF $(3.0 \mathrm{~mL})$ were mixed in a glass pressure tube. Then, the sealed tube was placed in an oven at 105 ${ }^{\circ} \mathrm{C}$ for $48 \mathrm{~h}$. After $48 \mathrm{~h}$, the tube was removed from the oven and allowed to attain room temperature. The crystals were coated in Paratone oil and mounted on a MiTeGen loop for data collection. They were flash frozen in the liquid nitrogen gaseous stream of an Oxford cryosystem. The diffraction data were measured on the RigakuOD MX-007HF SynergyR X-ray diffractometer. The data were collected with $\mathrm{CuK} \alpha 1 \lambda=1.54184 \AA$. The crystal was kept at 100K. Data were collected as $\varphi$ and $\omega$ scans of $1^{\circ}$ frames with CrysAlisPro v40. The data were 
integrated and reduced using Crys AlisPro v40. ${ }^{\mathrm{S3}} \mathrm{A}$ multi-scan absorption correction was applied. The structure was solved by direct methods using SHELXT-2016/4 as implemented in Olex2. ${ }^{\mathrm{S}}$ The structure as fully refined with SHELXL-2016/4. ${ }^{\text {S5 }}$ All non-hydrogen atoms were refined with anisotropic displacement coefficients. Hydrogen atoms were placed in calculated positions and assigned isotropic displacement coefficients, and their coordinates were allowed to ride on the respective carbon atoms. The SQUEEZE protocol of Platon was used. ${ }^{\text {S6 }}$ 


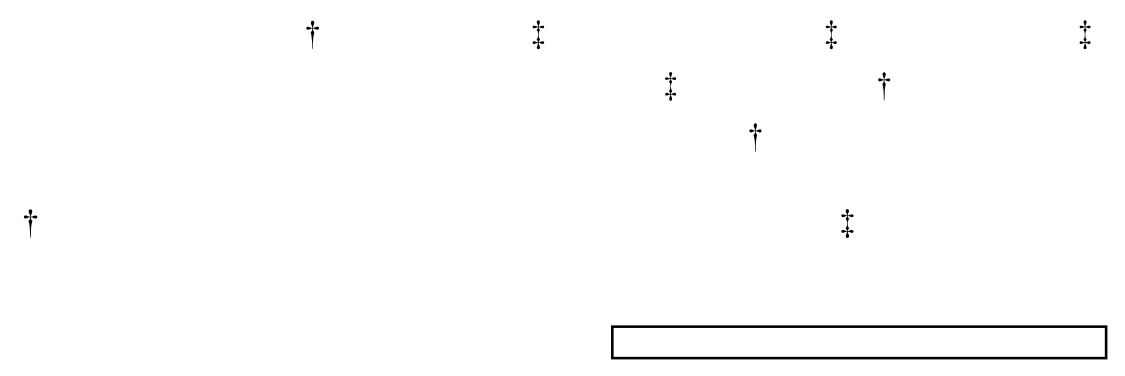

For details, see Table S1 and CCDC 2020813. 
Table S1. Crystal data and structure refinement of Ni-AdDB'.

Formula

Formula weight

Temperature

Wavelength

Crystal system

Space group

Unit cell dimensions

Volume

Z

Density (calc)

Absorption coefficient

F (000)

Crystal size

Theta range

Index range

Reflections collected

$R_{\text {int }}$
$\mathrm{C}_{62} \mathrm{H}_{52} \mathrm{NiN}_{4} \mathrm{O}_{2}$

$943.78(\mathrm{~g} / \mathrm{mol})$

$100 \mathrm{~K}$

$1.54184 \AA$

Hexagonal

P622

$\mathrm{a}=25.895(2) \AA \quad \alpha=90^{\circ}$

$\mathrm{b}=25.895(2) \AA \quad \beta=90^{\circ}$

$c=17.858(1) \AA \quad \gamma=120^{\circ}$

$10370.4(16) \AA^{3}$

6

$0.907 \mathrm{mg} / \mathrm{m}^{-3}$

$0.675 \mathrm{~mm}^{-1}$

2976

$0.020 \mathrm{~mm} \times 0.010 \mathrm{~mm} \times 0.010 \mathrm{~mm}$

$3.163^{\circ}$ to $58.92^{\circ}$

$-28 \leq h \leq 23,-28 \leq k \leq 7,-8 \leq l \leq 19$

20489 (4970)

0.0791 
Flack parameter

Completeness $(\%)$

Data/restraints/parameter

Goodness-of-fit on $F^{2}$

Final $R[I>2 \sigma(\mathrm{I})]$

$R$ (all data)

Largest diff peak and hole
$0.05(8)$

99.4

4970/83/313

0.899

$R_{1}=0.0910, \mathrm{w} R_{2}=0.2321$

$R_{1}=0.1339, \mathrm{w} R_{2}=0.2567$

$0.409 \mathrm{e} \cdot \AA^{-3}$ and $-0.309 \mathrm{e} \cdot \AA^{-3}$

Powder X-Ray Diffraction. Diffraction measurements were carried out in reflection geometry using a TTRAX III (Rigaku, Japan) diffractometer equipped with a rotating $\mathrm{Cu}$ anode operating at $50 \mathrm{kV}$ and $200 \mathrm{~mA}$. A bent graphite monochromator and a scintillation detector were aligned in the diffracted beam. $\theta / 2 \theta$ scans were performed under specular conditions in the Bragg-Brentano mode with variable slits. The specimens were dispersed on zero background silicon plates and kept under open air to let it dry. The samples were scanned from 3 to 25 degrees with step size of 0.025 degrees and scan speed of 0.5 degree per minute. The data analysis was performed using Jade Pro software (Materials Data, Inc.) where peak positions and widths were determined by a selfconsistent profile-fitting procedure. Unit cell parameter was obtained by Whole Pattern Fitting modules. Structural characteristics were obtained using a Whole Pattern Fitting (Jade module), the Pawley method, which uses a diffraction model to fit the measured pattern using non-linear leastsquare optimization. The coherent scattering length and micro-strains were estimated using the Williamson - Hall method.

Cryo-Scanning Electron Diffraction (SED) of Ni-AdDB. A suspension $(10 \mu \mathrm{L})$ of as synthesized crystals was dispersed on lacey carbon support on aluminium grids for TEM analysis. SED data were obtained in a double aberration-corrected Themis-Z microscope (Thermo Fisher Scientific Electron Microscopy Solutions, Hillsboro, USA) equipped with a high-brightness FEG at an acceleration voltage of $300 \mathrm{kV}$. For the diffraction recording an electron probe with a convergence angle of 0.2 mrad was adjusted in STEM microprobe mode and further defocussed 
by typically 5-10 $\mu \mathrm{m}$ to reduce probe size and electron flux. A primary beam current of less than $10 \mathrm{pA}$ was used. The total exposure was less than $1 \mathrm{e} / \AA^{2}$. The EMPAD (electron microscope pixel array detector) allowed rapid data collection of the entire unsaturated diffraction pattern with a pixel dwell time of $1 \mathrm{~ms}$ for each pattern. Typically, diffraction patterns were acquired over a raster of $256 \times 256$ pixels.

Electron Tomography. Tilt-series for electron tomography were acquired in the Themis- $\mathrm{Z}$ microscope in STEM mode with a probe semiconvergence angle of $8 \mathrm{mrad}$ on a Thermo Fisher Scientific bright-field detector. Tilt series were recorded with a tilt increment of 2 degrees in a tilt range between -60 degrees to +60 degrees. The tomogram was reconstructed using custom written software for image alignment and SIRT processing.

Quantification of Brainy Crystals. The crystals were imaged using scanning electron microscopy (SEM) at different time-points. Overall area, and then the area and shape of the domains were quantified using higher magnification, SEM images or AFM images. To measure the area of crystals the crystals were segmented from SEM images using StarDist, a deep learning-based detection method for microscopy images with star-convex shape priors. We used the provided versatile model which was pre-trained on fluorescent nuclei and worked surprisingly well on the SEM images. Crystals on the border of the image were discarded as well as some false-detected objects based on their size and standard deviation of their intensity. Some additional segmentation errors were manually corrected. We implemented this workflow as a Fiji macro. To quantify the detailed shape of domains on the surface of the crystals, higher magnification SEM and AFM images were used. In both cases the desired regions of analysis were manually selected. For the SEM images the domains were segmented using the Ilastik Boundary based segmentation with Multicut workflow. This workflow allows segmenting images based on boundary information. Given a boundary probability map, it breaks the image up into superpixels and then merges them to recover segments limited by closed surfaces. We trained a classifier for the boundary probability map and an edge classifier, in Ilastik and applied them to all the images in batch. A Fiji macro was written to process the candidate domains. The area, and the circularity and solidity shape features of domains that were completely included within the selected analysis regions were measured. Further manual correction was done to fix some segmentation errors. All the measurements were recalculated using the corrected segments. The macro supports this through an Update mode. The 
domains in AFM images were segmented by inverting the image, applying tubeness filter $(\sigma=10$ $\mathrm{nm}$ ), followed by Morphological Segmentation from the MorphoLibJ plugin (tolerance = 1). Small segments were discarded, and the remaining segments were filled and extended so that they touch each other using CLIJ plugin. Further processing was identical to the one used for the SEM images: include only domains completely within selected analysis regions. Quantify area, solidity and circularity, and optional manual correction. The same Fiji macro supports either SEM or AFM quantification and is available at the Github page. ${ }^{24}$ All the images were scaled and cropped prior to analysis. Both circularity and solidity equal one for a perfect circle and have lower values for other shapes. Circularity is a common compactness measure. It is the ratio of the area of the segment to the area of a circle (the most compact shape) having the same perimeter. As the value approaches zero, it indicates an increasingly elongated shape. Solidity is the area of a segment divided by its convex area (the imaginary convex hull around it). It is therefore an indicator of how "ruffled" the border of the segment is or how many (not quantitatively) concave cavities are on the surface of the segment. 

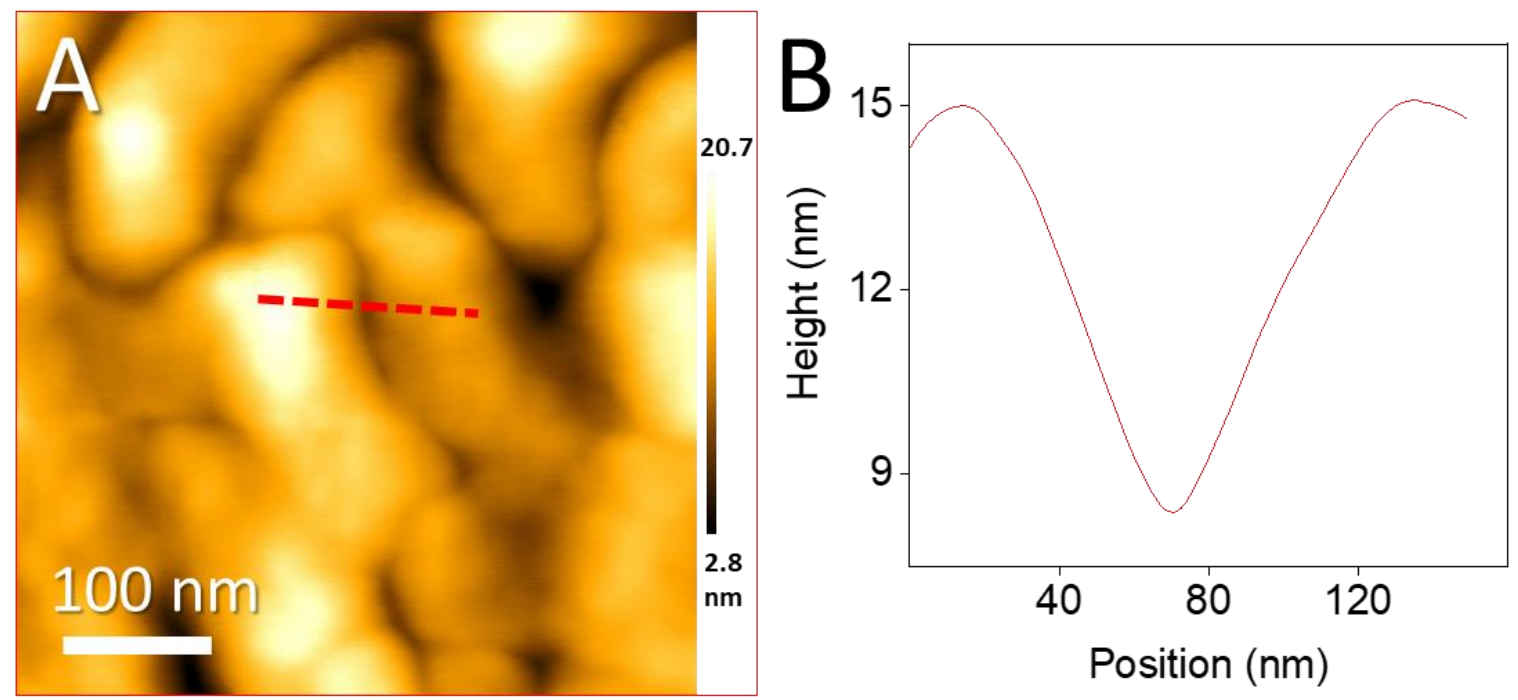

Figure S1. Surface details of a Ni-AdDB particle. (A) High resolution AFM topographic image of Ni-AdDB. (B) Height profile line as indicated in A. 

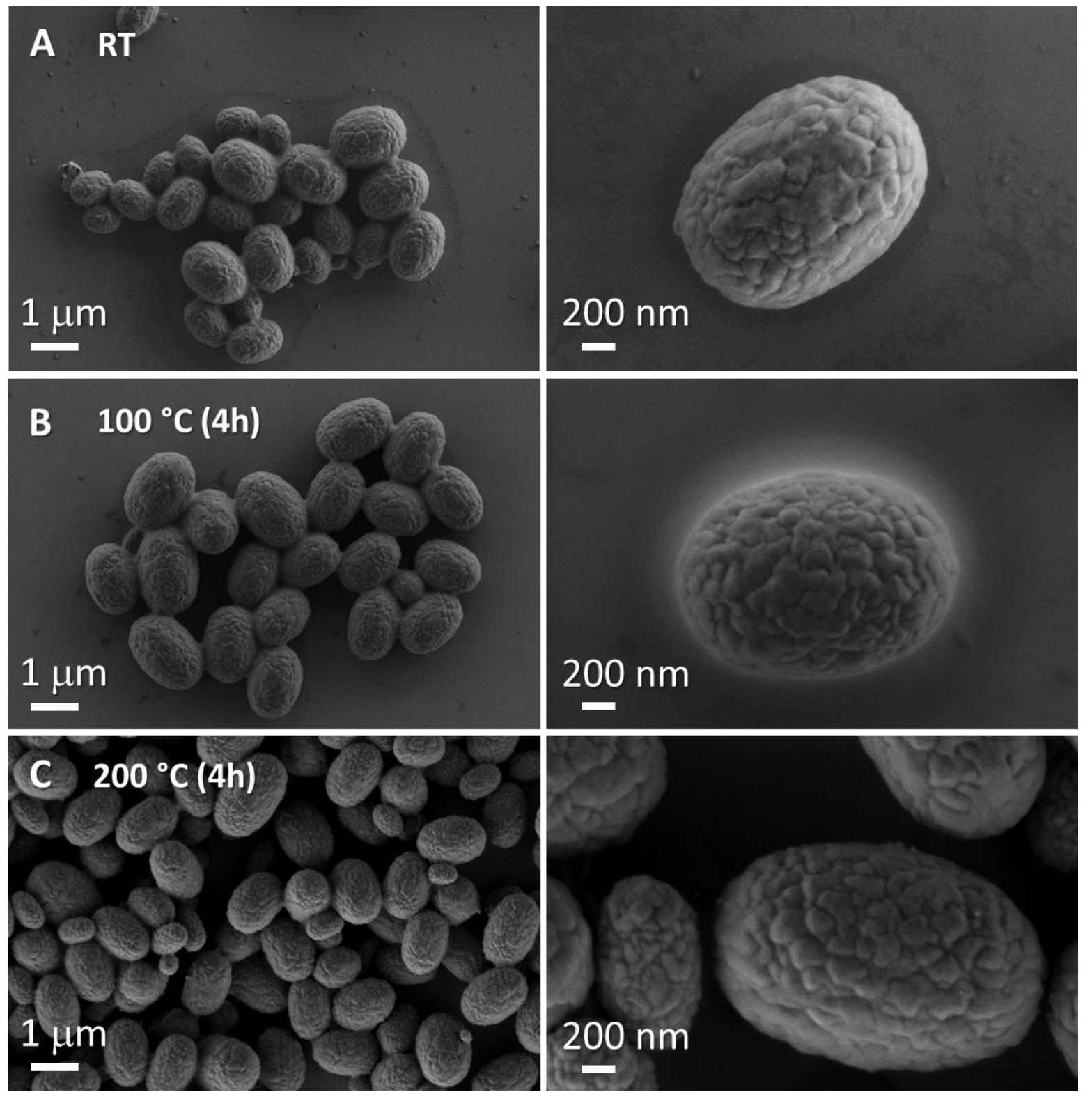

Figure S2. Scanning electron microscopy (SEM) images before and after heating in an oven for $4 \mathrm{~h}$ in air shows the morphological stability of Ni-AdDB. (A) Ni-AdDB at room temperature, (B) $100{ }^{\circ} \mathrm{C}$ and (C) $200{ }^{\circ} \mathrm{C}$. 

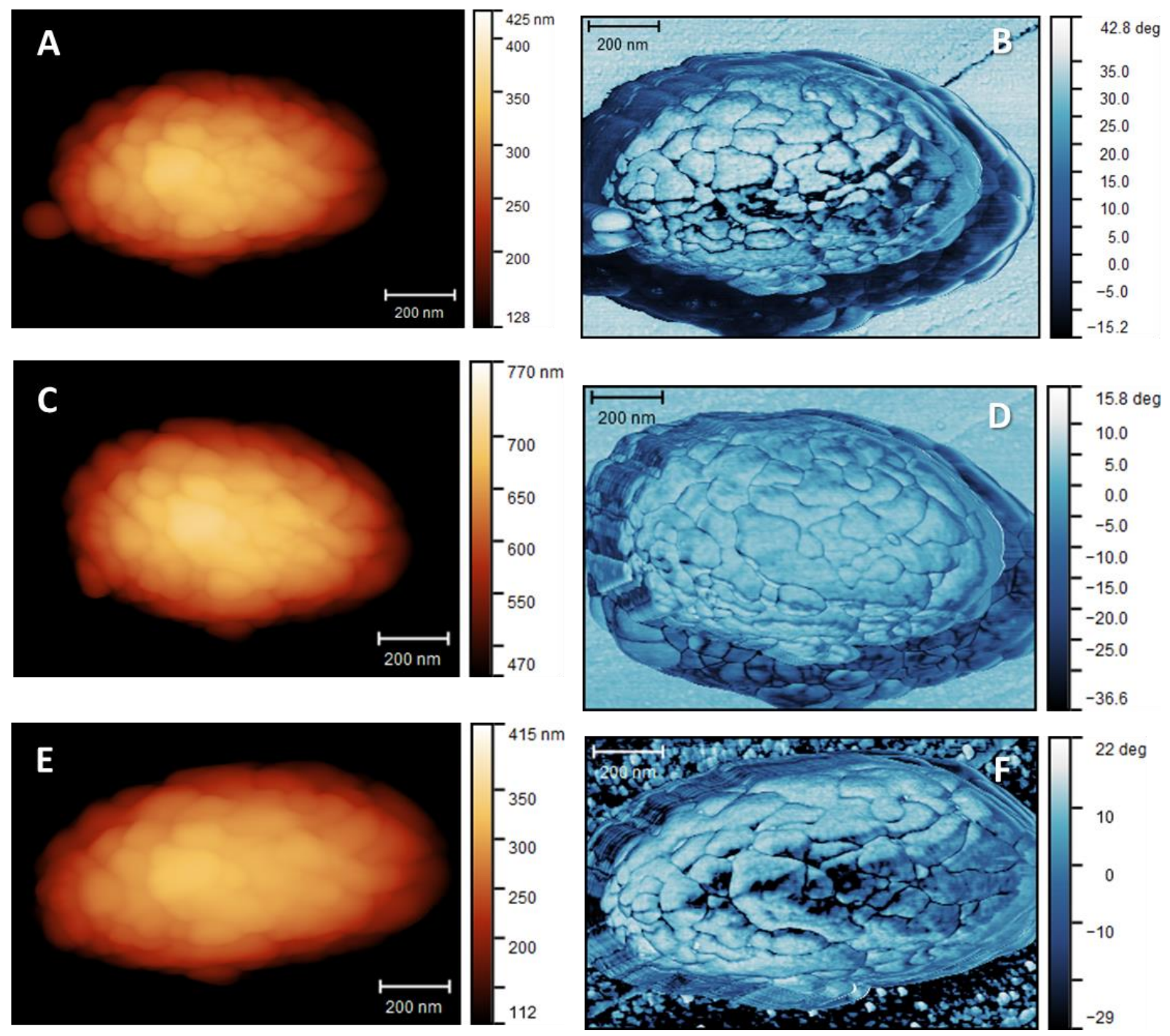

Figure S3. AFM topographic images and phase images of Ni-AdDB after heating in air for 4 h. (A, C, E) Phase images at room temperature, after heating at $100{ }^{\circ} \mathrm{C}$ and $200{ }^{\circ} \mathrm{C}$ respectively. (B, D, F) Topography images corresponding to A, C and E respectively. 


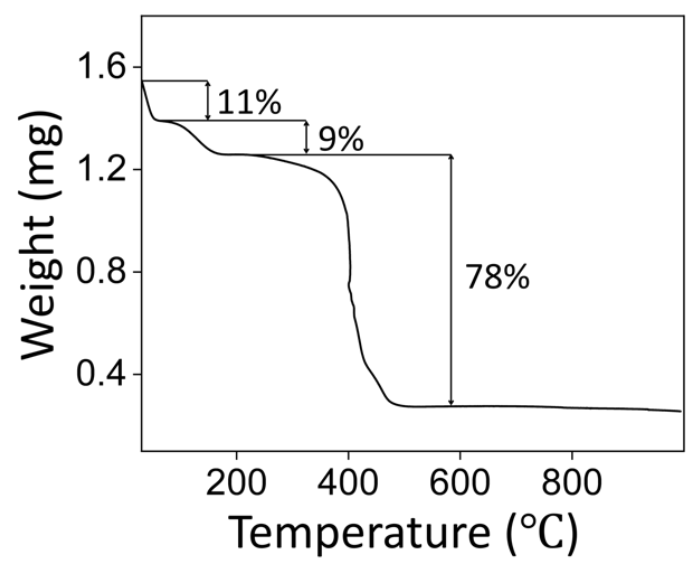

Figure S4. Thermal stability of Ni-AdDB using thermogravimetric analysis (TGA). Heating rate: $10^{\circ} \mathrm{C} / \mathrm{min}$, range: $25^{\circ} \mathrm{C}$ to $1000^{\circ} \mathrm{C}$ under a stream of air.

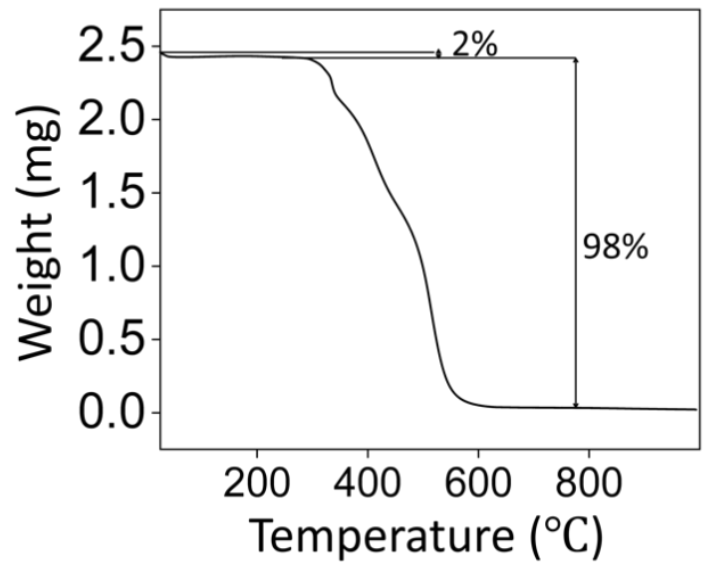

Figure S5. Thermal stability of AdDB using thermogravimetric analysis (TGA). Heating rate: $10{ }^{\circ} \mathrm{C} / \mathrm{min}$, range: $25^{\circ} \mathrm{C}$ to $1000{ }^{\circ} \mathrm{C}$ under a stream of air. 


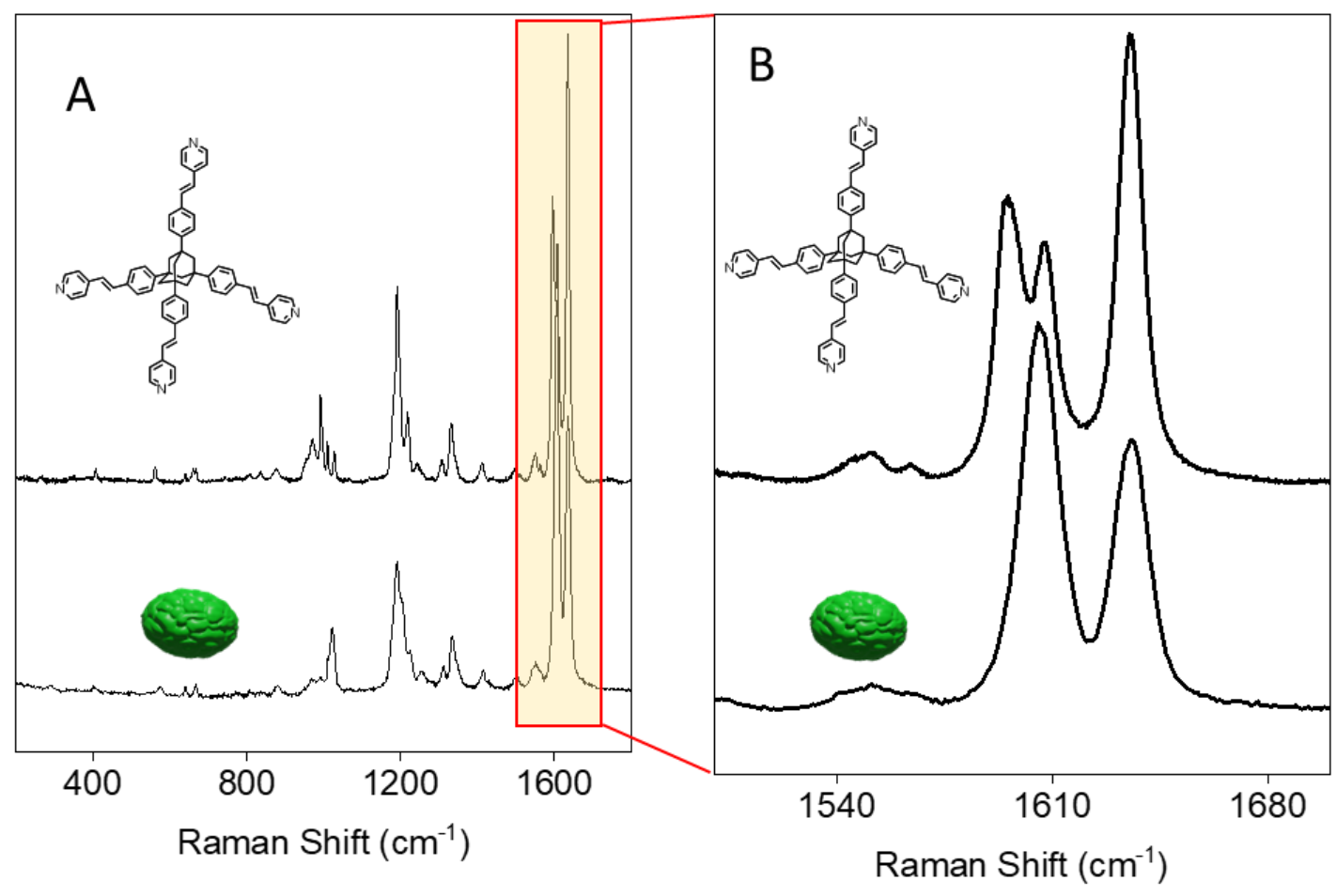

Figure S6. Micro Raman spectra. (A) Micro Raman spectra of AdDB (top) and Ni-AdDB (bottom) after 2 days of solvothermal. (B) Zoom-in of spectrum A in the range from $1400 \mathrm{~cm}^{-1}$ to $1700 \mathrm{~cm}^{-1}$.

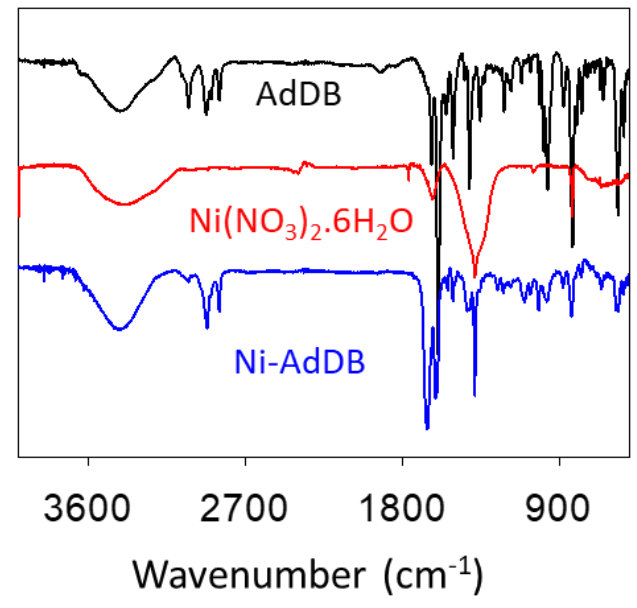

Figure S7. Bulk sample analysis by Infrared spectroscopy using a $\mathrm{KBr}$ pellet of AdDB (black), $\mathrm{Ni}\left(\mathrm{NO}_{3}\right)_{2} \cdot 6 \mathrm{H}_{2} \mathrm{O}$ (red), and Ni-AdDB (blue). 


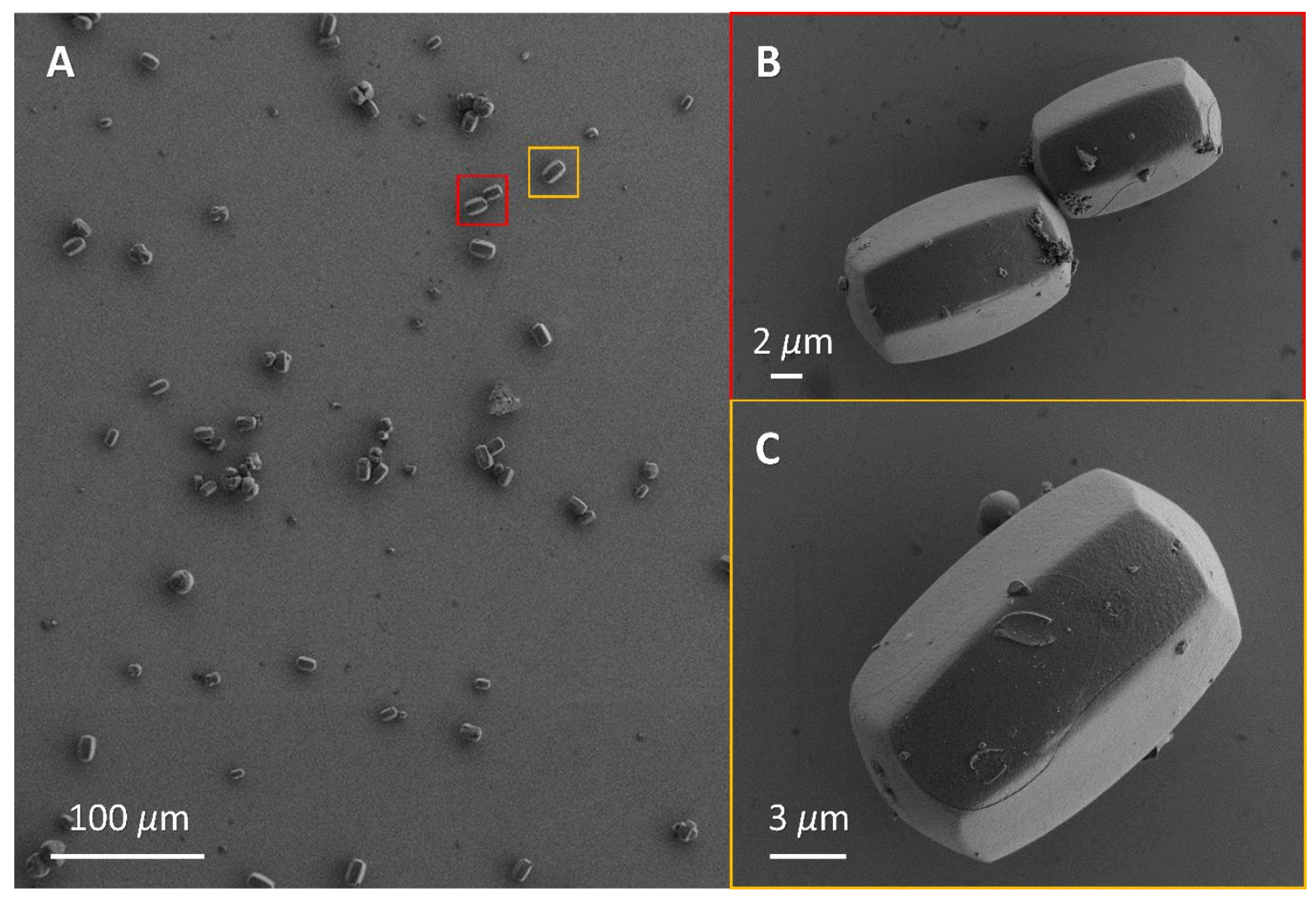

Figure S8. Scanning Electron Microscopy (SEM) of Ni-AdDB'. A) Low magnification SEM image. (B-C) High magnification SEM images marked in (A).
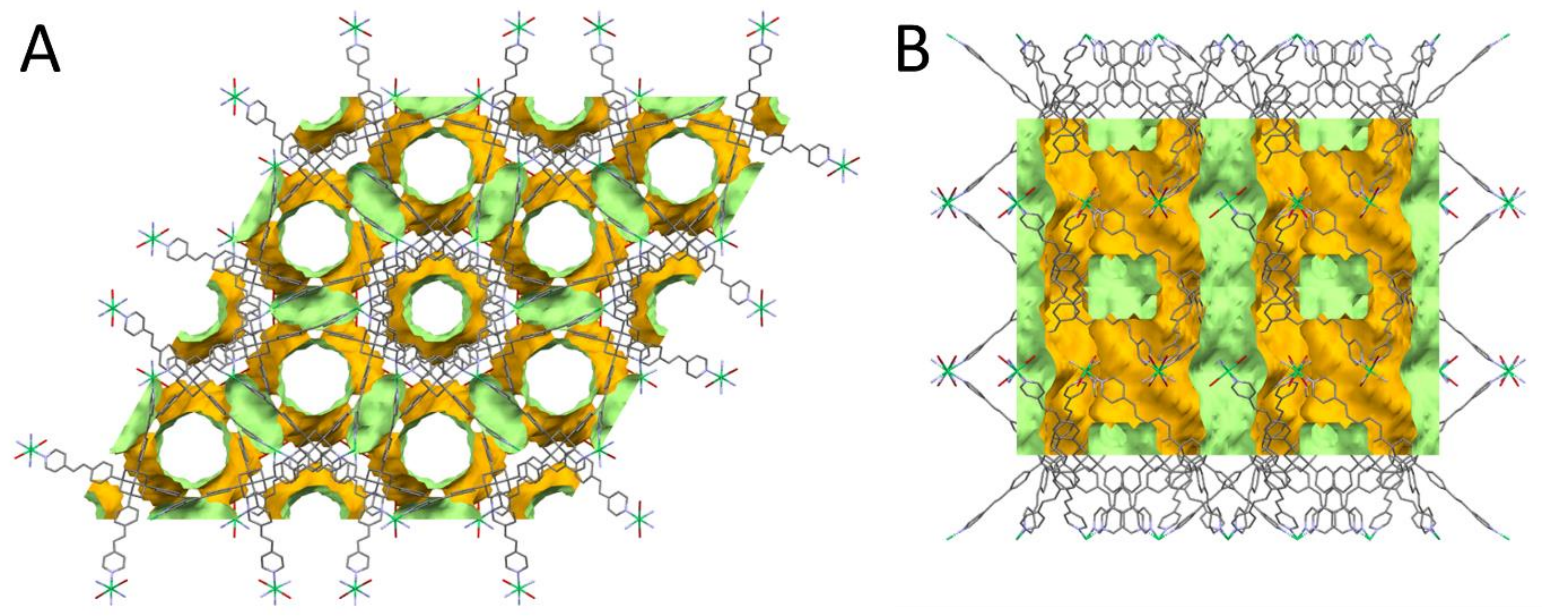

Figure S9. Connolly surface representation of the crystal structure. (A,B) Presentation along $\mathrm{c}$ and a axes respectively. Crystal structure shows $38 \%$ void (empty space) after using $1.2 \AA$ probe radius and $\sim 0.7 \AA$ grid spacing. 

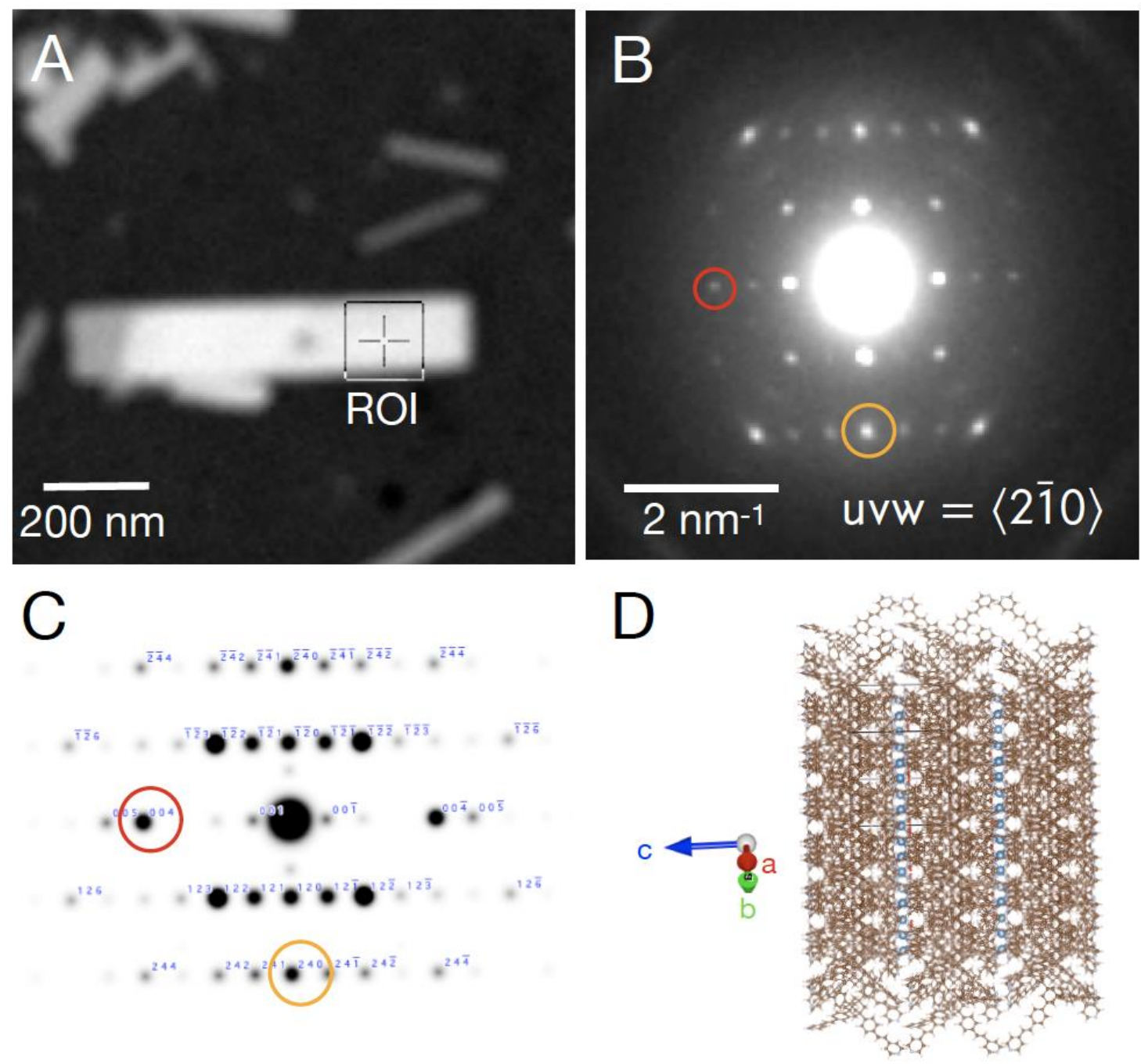

Figure S10. Scanning electron diffraction (SED) obtained from plates formed after 2 min. reaction time. (A) Virtual annular dark field (ADF) image of a plate. (B) Diffraction pattern obtained from a marked region of interest (ROI) in A. (C) Simulated diffraction pattern from single crystal data and (D) view of crystallographic packing of Ni-AdDB' in $\langle 2 \overline{1} 0\rangle$ view direction. 

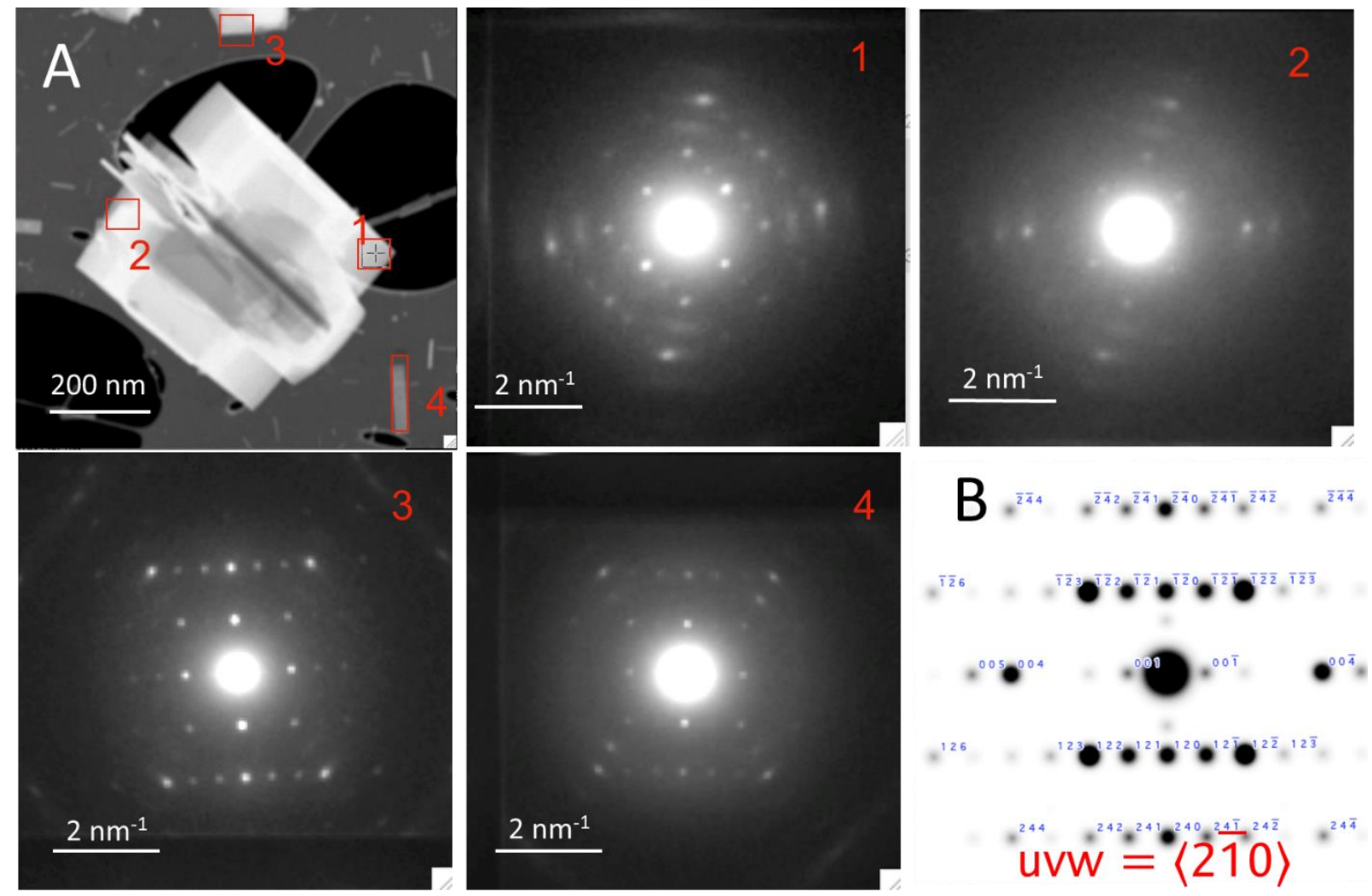

Figure S11. Nano beam electron diffraction obtained from fused plates. (A) Virtual annular dark field (ADF) image of a plate-like structure and electron diffractions of marked regions 1-4 are shown in panel 1-4. (B) Simulated diffraction pattern from Ni-AdDB' single crystal along $\langle 2 \overline{1} 0\rangle$ zone axis. 


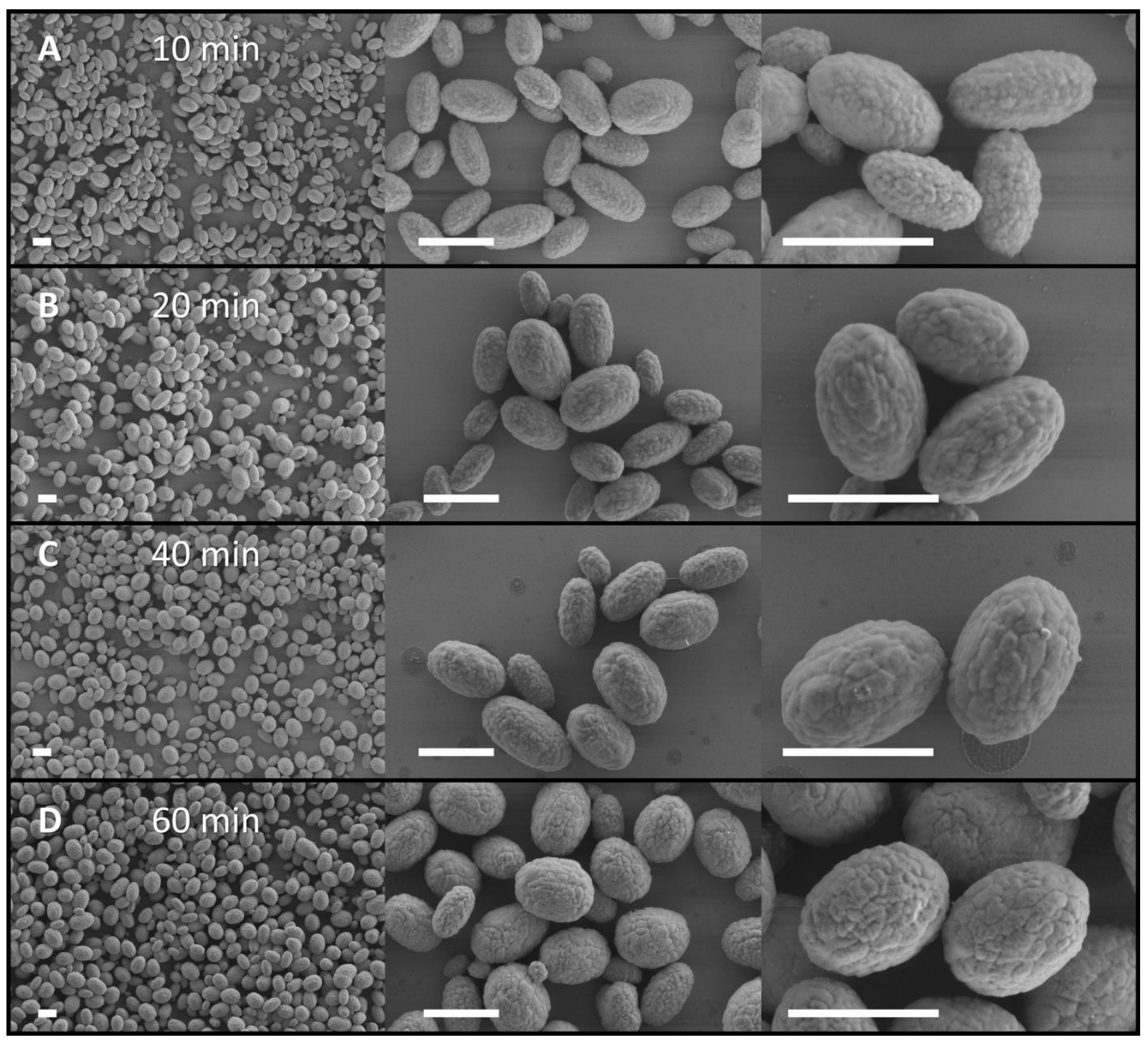

Figure S12. Ex situ scanning electron microscopy (SEM) images at different times during synthesis. (A-D) SEM images after $10 \mathrm{~min}, 20 \mathrm{~min}, 40 \mathrm{~min}$ and $60 \mathrm{~min}$ of solvothermal synthesis, respectively. Scale bar $=1 \mu \mathrm{m}$. 


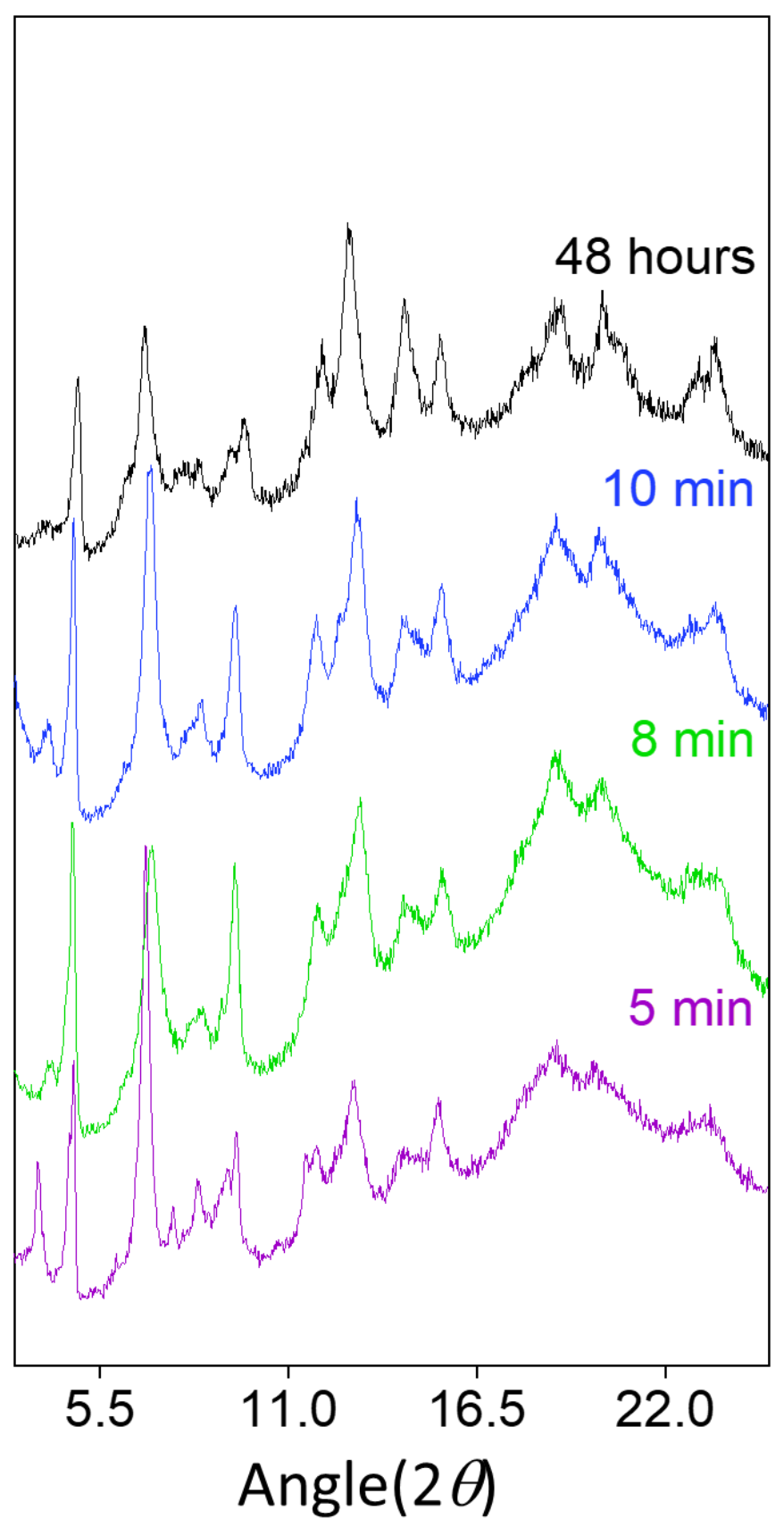

Figure S13. Powder X ray diffraction pattern of Ni-AdDB at different reaction times. 


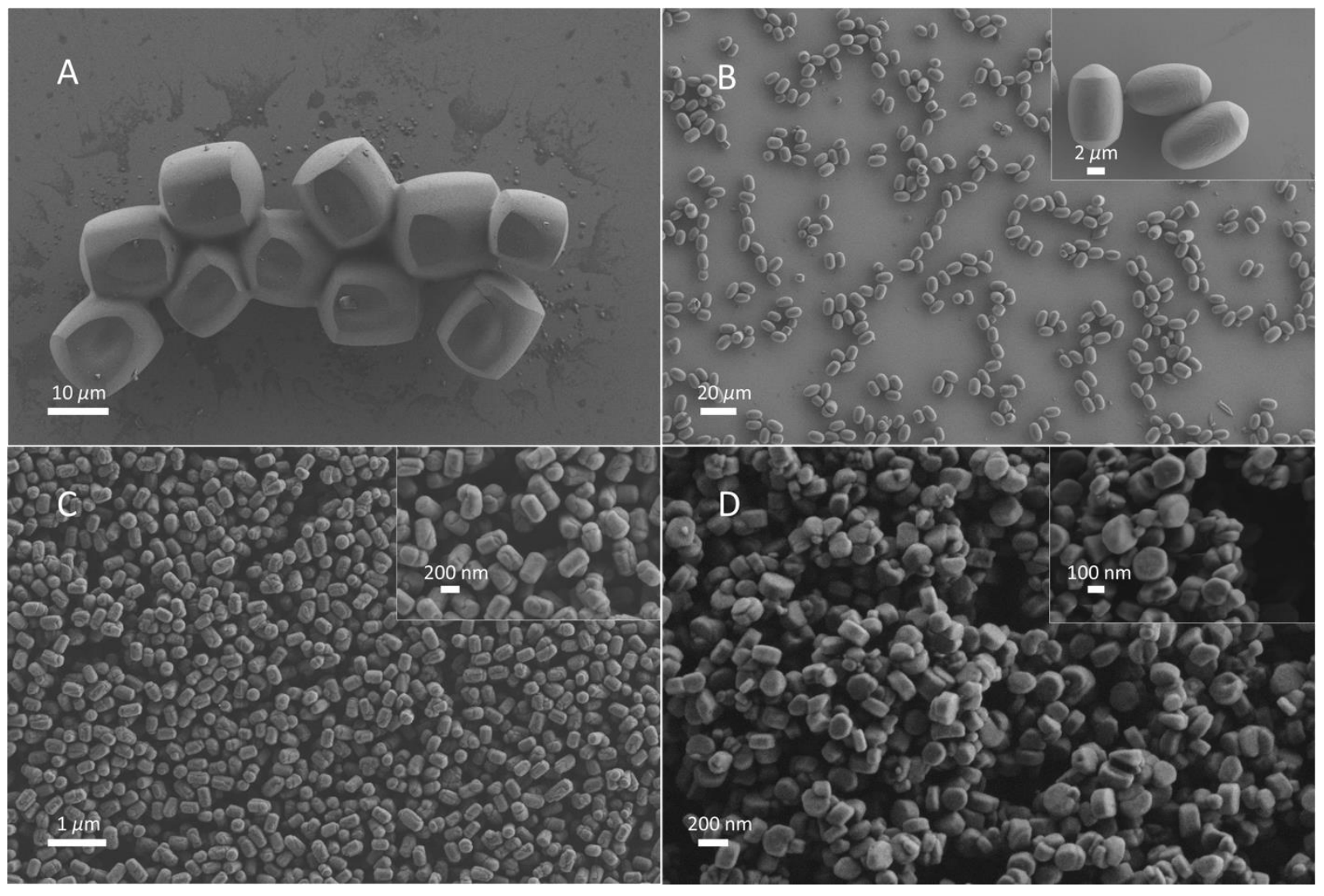

Figure S14. Scanning electron microscopy (SEM) images at different supersaturation levels. $\mathrm{Ni}\left(\mathrm{NO}_{3}\right)_{2} .6 \mathrm{H}_{2} \mathrm{O}$ and AdDB (ratio 2:1) in $4 \mathrm{~mL}$ of solvent (3 mL DMF and $1 \mathrm{~mL}$ chloroform). (A) $\mathrm{Ni}\left(\mathrm{NO}_{3}\right)_{2} .6 \mathrm{H}_{2} \mathrm{O}: 0.7 \mathrm{mg}$, AdDB: $1 \mathrm{mg}$, (B) $\mathrm{Ni}\left(\mathrm{NO}_{3}\right)_{2} .6 \mathrm{H}_{2} \mathrm{O}: 1.7 \mathrm{mg}$, AdDB: $2.5 \mathrm{mg}$, (C) $\mathrm{Ni}\left(\mathrm{NO}_{3}\right)_{2} .6 \mathrm{H}_{2} \mathrm{O}: 6.8 \mathrm{mg}$, AdDB: $10 \mathrm{mg}$ and (D) $\mathrm{Ni}\left(\mathrm{NO}_{3}\right)_{2} .6 \mathrm{H}_{2} \mathrm{O}: 17 \mathrm{mg}$, AdDB: $25 \mathrm{mg}$. 


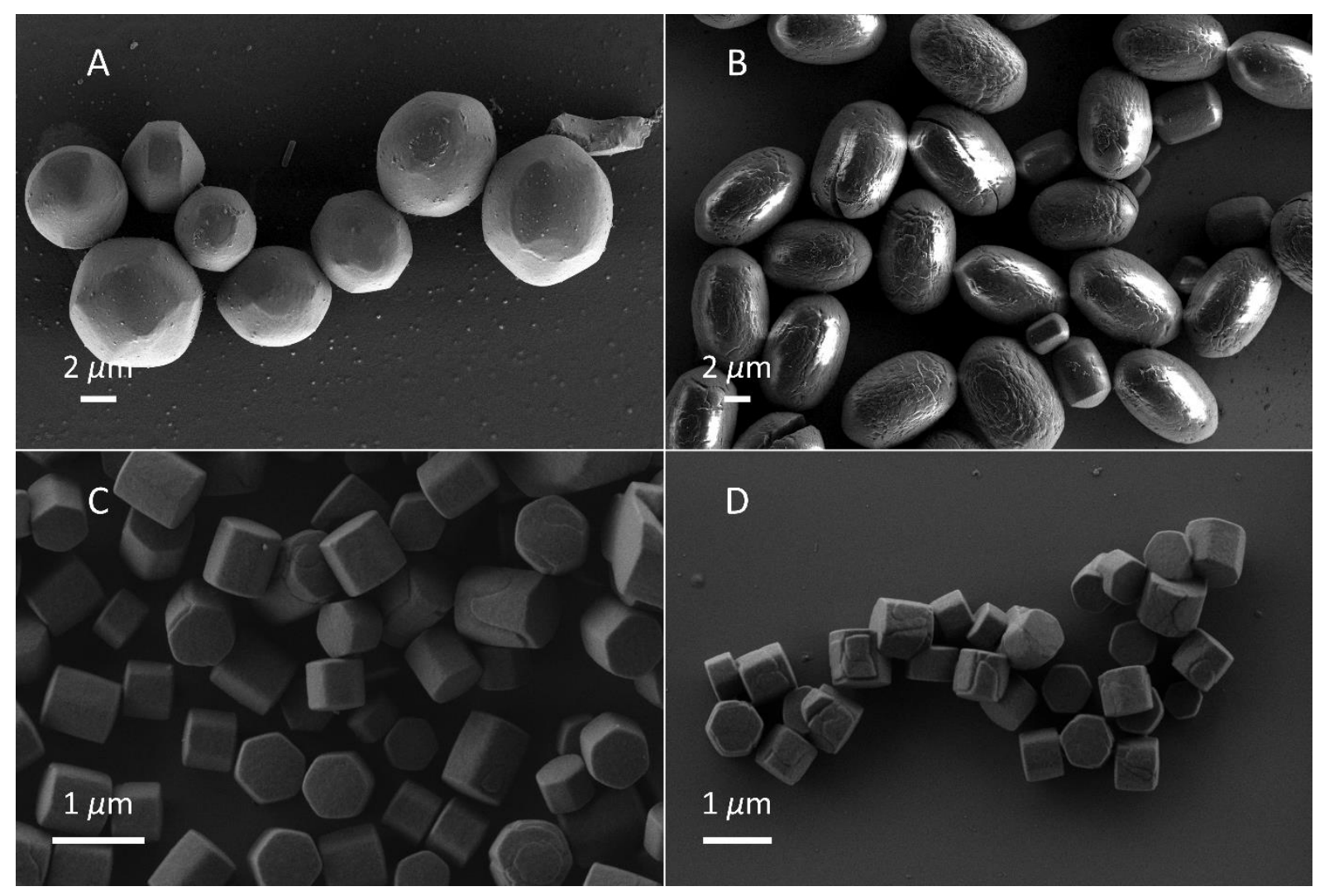

Figure S15. Scanning electron microscopy (SEM) images at different stoichiometric ratios between $\mathrm{Ni}\left(\mathrm{NO}_{3}\right)_{2} \cdot 6 \mathrm{H}_{2} \mathrm{O}$ and AdDB: $0.3: 1,1.5: 1,4: 1$ to $6: 1 \mathrm{in} 4 \mathrm{~mL}$ solvent (3 $\mathrm{mL} \mathrm{DMF}$ and 1 mL chloroform). (A) $\mathrm{Ni}_{(}\left(\mathrm{NO}_{3}\right)_{2} .6 \mathrm{H}_{2} \mathrm{O}: 0.5 \mathrm{mg}$, AdDB: $5.0 \mathrm{mg}$ (B) Ni( $\left.\mathrm{NO}_{3}\right)_{2} .6 \mathrm{H}_{2} \mathrm{O}: 2.6 \mathrm{mg}$, AdDB: $5.0 \mathrm{mg},(\mathrm{C}) \mathrm{Ni}\left(\mathrm{NO}_{3}\right)_{2} .6 \mathrm{H}_{2} \mathrm{O}: 6.8 \mathrm{mg}$, AdDB: $5.0 \mathrm{mg}$ and (D) $\mathrm{Ni}\left(\mathrm{NO}_{3}\right)_{2} .6 \mathrm{H}_{2} \mathrm{O}: 10 \mathrm{mg}$, AdDB: 5.0 $\mathrm{mg}$. 


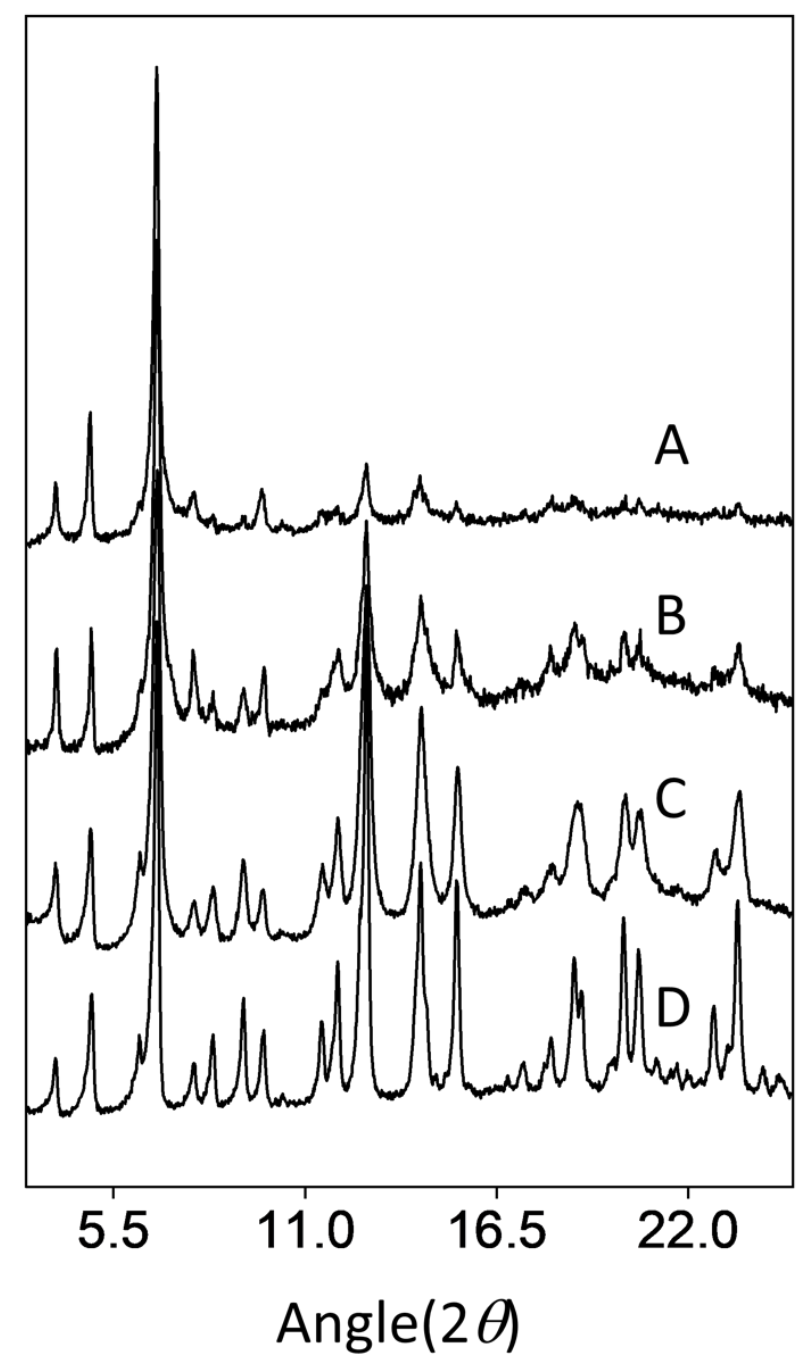

Figure S16. PXRD spectra of crystals obtained using different supersaturation of $\mathrm{Ni}\left(\mathrm{NO}_{3}\right)_{2} \cdot 6 \mathrm{H}_{2} \mathrm{O}$ and AdDB. $\mathrm{Ni}\left(\mathrm{NO}_{3}\right)_{2} \cdot 6 \mathrm{H}_{2} \mathrm{O}$ and AdDB (ratio 2:1) in $4 \mathrm{~mL}$ of solvent $(3 \mathrm{~mL} \mathrm{DMF}$ and $1 \mathrm{~mL}$ chloroform). (A) $\mathrm{Ni}\left(\mathrm{NO}_{3}\right)_{2} \cdot 6 \mathrm{H}_{2} \mathrm{O}: 0.7 \mathrm{mg}$, AdDB: $1 \mathrm{mg}$ (B) $\mathrm{Ni}\left(\mathrm{NO}_{3}\right)_{2} \cdot 6 \mathrm{H}_{2} \mathrm{O}: 1.7 \mathrm{mg}$, AdDB: 2.5 $\mathrm{mg},(\mathrm{C}) \mathrm{Ni}\left(\mathrm{NO}_{3}\right)_{2} .6 \mathrm{H}_{2} \mathrm{O}: 6.8 \mathrm{mg}$, AdDB: $10 \mathrm{mg}$ and (D) $\mathrm{Ni}\left(\mathrm{NO}_{3}\right)_{2} .6 \mathrm{H}_{2} \mathrm{O}: 17 \mathrm{mg}$, AdDB: $25 \mathrm{mg}$. 


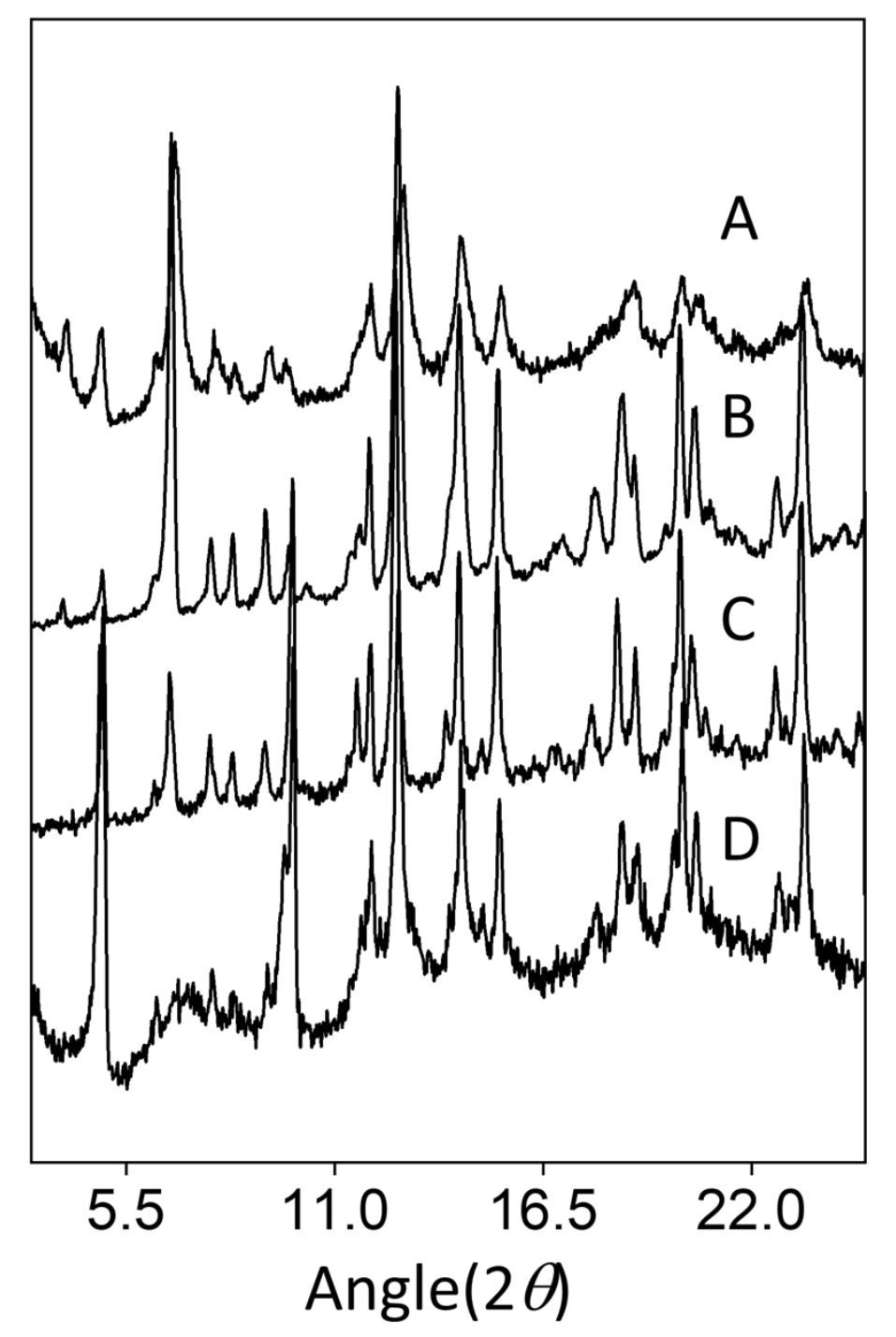

Figure S17. PXRD spectra of crystals obtained using different stoichiometric ratios between $\mathrm{Ni}\left(\mathrm{NO}_{3}\right)_{2} \cdot 6 \mathrm{H}_{2} \mathrm{O}$ and AdDB: from $0.3: 1,1.5: 1,4: 1$ to $6: 1$ in $4 \mathrm{~mL}$ solvent $(3 \mathrm{~mL} \mathrm{DMF}$ and $1 \mathrm{~mL}$ chloroform). (A) $\mathrm{Ni}\left(\mathrm{NO}_{3}\right)_{2} .6 \mathrm{H}_{2} \mathrm{O}: 0.5 \mathrm{mg}$, AdDB: $5.0 \mathrm{mg}$ (B) Ni(NO$\left.)_{2}\right)_{2} 6 \mathrm{H}_{2} \mathrm{O}: 2.6 \mathrm{mg}$, AdDB: 5.0 $\mathrm{mg}$, (C) $\mathrm{Ni}\left(\mathrm{NO}_{3}\right)_{2} .6 \mathrm{H}_{2} \mathrm{O}: 6.8 \mathrm{mg}$, AdDB: $5.0 \mathrm{mg}$ and (D) $\mathrm{Ni}\left(\mathrm{NO}_{3}\right)_{2} .6 \mathrm{H}_{2} \mathrm{O}: 10 \mathrm{mg}$, AdDB: $5.0 \mathrm{mg}$. 

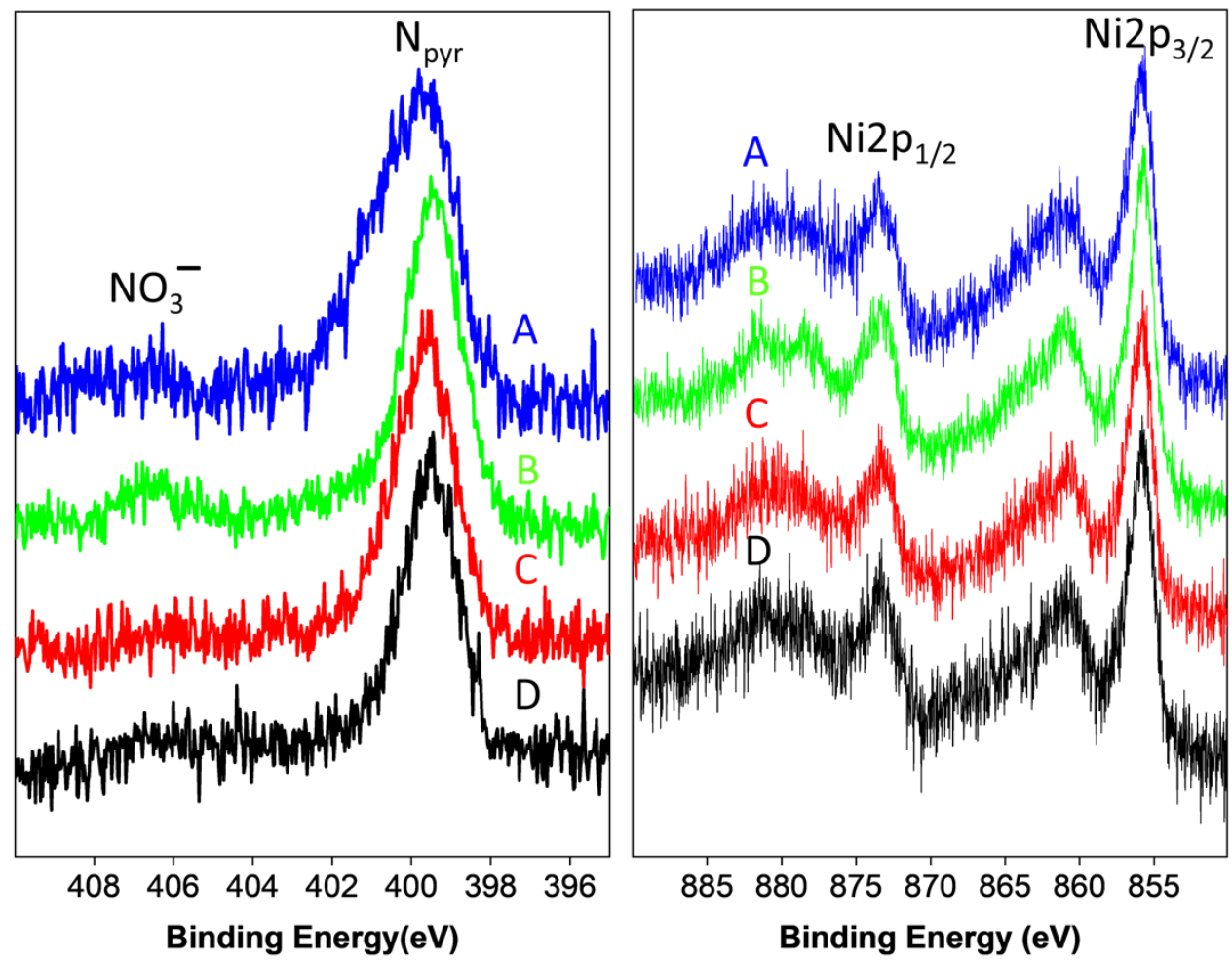

Figure S18. Building block ratios on crystal surface at different stoichiometry using X-ray photoelectron spectroscopy. XPS measurement (left panel: $\mathrm{N}_{\text {pyr }}$ and $\mathrm{NO}_{3}{ }^{-}$peaks and right panel: $\mathrm{Ni}^{2+} 2 \mathrm{p}_{3 / 2}$ and $2 \mathrm{p}_{1 / 2}$ peaks) from bottom to top is taken for following $\mathrm{Ni}\left(\mathrm{NO}_{3}\right)_{2} \cdot 6 \mathrm{H}_{2} \mathrm{O}$ and AdDB ratio: 0.3:1, 1:1. 2:1 and 6:1 respectively. (A) $\mathrm{Ni}\left(\mathrm{NO}_{3}\right)_{2} \cdot 6 \mathrm{H}_{2} \mathrm{O}: 0.5 \mathrm{mg}$, AdDB: 5.0 mg, (B) $\mathrm{Ni}\left(\mathrm{NO}_{3}\right)_{2} .6 \mathrm{H}_{2} \mathrm{O}: 1.7 \mathrm{mg}$, AdDB: $5.0 \mathrm{mg}$, (C) $\mathrm{Ni}\left(\mathrm{NO}_{3}\right)_{2} .6 \mathrm{H}_{2} \mathrm{O}: 3.4 \mathrm{mg}$, AdDB: 5.0 $\mathrm{mg}$, and (D) $\mathrm{Ni}\left(\mathrm{NO}_{3}\right)_{2} \cdot 6 \mathrm{H}_{2} \mathrm{O}: 10 \mathrm{mg}$, AdDB: $5.0 \mathrm{mg}$.

\section{References}

S1. Neumann, T., Jess, I., Nather, C. (2018). Crystal structures of (acetonitrile-kappa N)tris(pyridine-4-thioamide-kappa N)bis(thiocyanato-kappa N)cobalt(II) acetonitrile disolvate and tetrakis(pyridine-4-thioamide-kappa N)bis(thiocyanato-kappa N)nickel(II) methanol pentasolvate. Acta Crystallographica Section E-Crystallographic Communications, 74, 964.

S2. http://gwyddion.net/

S3. CrysAlisPro 40: Rigaku OD (2018). CrysAlis PRO. Rigaku Oxford Diffraction, Yarnton, England.

S4. Dolomanov, O. V., Bourhis, L. J., Gildea, R. J., Howard, J. A. K. and Puschmann, H. (2009). OLEX2: a complete structure solution, refinement and analysis program. J. Appl. Cryst. 42, 339-341. 
S5. Sheldrick, G. M. (2015). Crystal structure refinement with SHELXL. Acta Cryst. C71, 38.

S6 Spek, A. L. (2009). Structure validation in chemical crystallography. Acta Cryst. D65, $148-155$. 